\title{
Multiday maintenance of extracorporeal lungs using cross-circulation with conscious swine
}

\author{
Ahmed E. Hozain, MD, ${ }^{\text {a,b }}$ Yuliya Tipograf, MD, ${ }^{\text {,c }}$ Meghan R. Pinezich, MS, ${ }^{a}$ \\ Katherine M. Cunningham, BS, ${ }^{\mathrm{a}}$ Rachel Donocoff, DVM, ${ }^{\mathrm{d}}$ Dawn Queen, BA, ${ }^{\mathrm{e}}$ Kenmond Fung, MS, CCP, \\ Charles C. Marboe, MD, ${ }^{\mathrm{g}}$ Brandon A. Guenthart, MD, ${ }^{\mathrm{a}}$ John D. O'Neill, PhD, ${ }^{\mathrm{a}}$ \\ Gordana Vunjak-Novakovic, $\mathrm{PhD},{ }^{\mathrm{a}, \mathrm{h}}$ and Matthew Bacchetta, MD, MBA, MA ${ }^{\mathrm{a}, \mathrm{c}}$
}

\section{ABSTRACT}

Objectives: Lung remains the least-utilized solid organ for transplantation. Efforts to recover donor lungs with reversible injuries using ex vivo perfusion systems are limited to $<24$ hours of support. Here, we demonstrate the feasibility of extending normothermic extracorporeal lung support to 4 days using crosscirculation with conscious swine.

Methods: A swine behavioral training program and custom enclosure were developed to enable multiday cross-circulation between extracorporeal lungs and recipient swine. Lungs were ventilated and perfused in a normothermic chamber for 4 days. Longitudinal analyses of extracorporeal lungs (ie, functional assessments, multiscale imaging, cytokine quantification, and cellular assays) and recipient swine (eg, vital signs and blood and tissue analyses) were performed.

Results: Throughout 4 days of normothermic support, extracorporeal lung function was maintained (arterial oxygen tension/inspired oxygen fraction $>400 \mathrm{~mm}$ $\mathrm{Hg}$; compliance $>20 \mathrm{~mL} / \mathrm{cm} \mathrm{H}_{2} \mathrm{O}$ ), and recipient swine were hemodynamically stable (lactate $<3 \mathrm{mmol} / \mathrm{L} ; \mathrm{pH}, 7.42 \pm 0.05$ ). Radiography revealed wellaerated lower lobes and consolidation in upper lobes of extracorporeal lungs, and bronchoscopy showed healthy airways without edema or secretions. In bronchoalveolar lavage fluid, granulocyte-macrophage colony-stimulating factor, interleukin (IL) 4, IL-6, and IL-10 levels increased less than 6-fold, whereas interferon gamma, IL- $1 \alpha$, IL-1 $\beta$, IL-1ra, IL-2, IL-8, IL-12, IL-18, and tumor necrosis factor alpha levels decreased from baseline to day 4. Histologic evaluations confirmed an intact blood-gas barrier and outstanding preservation of airway and alveolar architecture. Cellular viability and metabolism in extracorporeal lungs were confirmed after 4 days.

Conclusions: We demonstrate feasibility of normothermic maintenance of extracorporeal lungs for 4 days by cross-circulation with conscious swine. Cross-

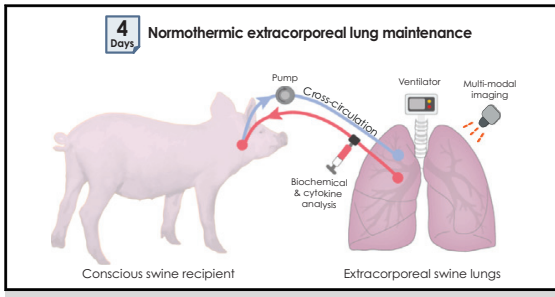

Multiday normothermic support system for extracorporeal lungs using cross-circulation.

\section{Central Message}

Normothermic support of extracorporeal lungs for 4 days is feasible using cross-circulation and could enhance recovery of donor lungs and enable methods to bioengineer lungs for transplantation.

\section{Perspective}

Conventional ex vivo lung perfusion systems offer limited time for recovery and therapeutic intervention in extracorporeal lungs. We demonstrate that normothermic preservation of extracorporeal lung tissue structure and respiratory function can be maintained for 4 days using cross-circulation. This system could serve as a platform for lung bioengineering and organ recovery and regeneration.

See Commentaries on pages 1654 and 1656. circulation approaches could support the recovery of damaged lungs and enable organ bioengineering to improve transplant outcomes. (J Thorac Cardiovasc Surg 2020;159:1640-53)

\footnotetext{
From the Departments of ${ }^{\mathrm{a}}$ Biomedical Engineering, ${ }^{\mathrm{b}}$ Surgery, ${ }^{\mathrm{f}} \mathrm{Clinical}$ Perfusion, ${ }^{\mathrm{g}}$ Pathology and Cell Biology, and ${ }^{\mathrm{h}}$ Medicine, ${ }^{\mathrm{e}}$ Vagelos College of Physicians and Surgeons, and ${ }^{\mathrm{d} I n s t i t u t e}$ of Comparative Medicine, Columbia University Medical Center, Columbia University, New York, NY; and ${ }^{\mathrm{c}}$ Departments of Thoracic and Cardiac Surgery, Vanderbilt University, Nashville, Tenn.

Supported by the National Institutes of Health (grant Nos. HL120046, HL134760, HL007854, HL143733, and EB027062), the Richard Bartlett Foundation, the Blavatnik Foundation, and the Mikati Foundation.

Read at the 99th Annual Meeting of The American Association for Thoracic Surgery, Toronto, Ontario, Canada, May 4-7, 2019.

Received for publication June 21, 2019; revisions received Sept 5, 2019; accepted for publication Sept 9, 2019; available ahead of print Nov 22, 2019.
}

Address for reprints: Matthew Bacchetta, MD, MBA, MA, Departments of Thoracic and Cardiac Surgery, Vanderbilt University, Nashville, TN 37232 (E-mail: matthew.bacchetta@vumc.org); and Gordana Vunjak-Novakovic, PhD, Department of Medicine, Columbia University Medical Center, Columbia University, New York, NY 10032 (E-mail: gv2131@columbia.edu). 0022-5223

Copyright $(2019$ by The American Association for Thoracic Surgery. Published by Elsevier Inc. This is an open access article under the CC BY-NC-ND license (http:// creativecommons.org/licenses/by-nc-nd/4.0/).

https://doi.org/10.1016/j.jtcvs.2019.09.121 


\section{Abbreviations and Acronyms}

$\mathrm{BAL}=$ bronchoalveolar lavage

$\mathrm{EVLP}=$ ex vivo lung perfusion

$\mathrm{IFN} \gamma=$ interferon gamma

IL $\quad=$ interleukin

$\mathrm{TNF} \alpha=$ tumor necrosis factor alpha

$\mathrm{XC}=$ cross-circulation

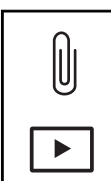

Scanning this QR code will

take you to the article title

page to access supplementary

information. To view the

AATS Annual Meeting Web-

cast, see the URL next to the

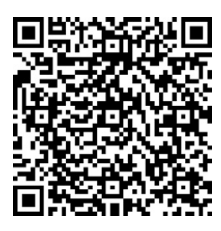

webcast thumbnail.

Lung transplantation, the only life-saving intervention for patients with end-stage lung disease, remains limited by the shortage of usable donor organs. Although the number of patients waiting to receive a lung transplant continues to rise, only $20 \%$ of donor lungs meet functional criteria for transplantation. ${ }^{1}$ This underuse of donor organs combined with the rising number of patients in need represents a major contributor to waitlist mortality. ${ }^{2,3}$

Many of the conditions that render donor lungs unacceptable for transplantation (eg, aspiration, infection, and pulmonary contusions) are potentially reversible, but conventional methods of donor lung preservation rely on nonphysiologic cold static ischemia and preclude endogenous repair and recovery. ${ }^{4,5} \mathrm{Ex}$ vivo lung perfusion (EVLP) aims to address these limitations by providing initially unacceptable donor lungs with physiologic conditions-normothermia, perfusion, ventilation-to recover function outside the body to a level acceptable for transplantation. ${ }^{6,7}$ Since the introduction of EVLP by Steen and colleagues in $2001,{ }^{8}$ EVLP platforms have demonstrated short-term support and recovery of marginal quality donor lungs in preclinical and clinical settings. ${ }^{8-11}$ However, EVLP has been unable to recover the majority of unusable donor lungs, likely due to the inability of an isolated single-organ support system to provide an appropriate physiologic milieu that enables endogenous repair.

Despite efforts to address the shortage of transplantable lungs, physiologic constraints limit lung perfusion and preservation times, and thus restrict opportunities for donor organ recovery and bioengineering. To overcome these limitations, our group previously used a swine model of cross-circulation (XC) to establish a lung support system that extended normothermic extracorporeal support to
36 hours and enabled statistically significant ex vivo recovery of severely injured lungs. ${ }^{12,13}$

The use of an XC system to achieve ex vivo lung recovery mimics the clinical setting where in situ recovery of marginal quality lungs is achieved in patients placed on extracorporeal membrane oxygenation support for several days after transplantation. ${ }^{14-16}$ Motivated by this clinical practice, we hypothesize that extending the duration of extracorporeal support from hours to days could not only enable the recovery of damaged lungs not currently salvageable using EVLP systems but also enable investigation of bioengineering strategies to improve or personalize organs before transplantation.

In this proof-of-feasibility study, we developed an extracorporeal lung support system capable of maintaining lungs for 4 days using XC (Figure 1). A notable difference between this study and our previous studies, wherein swine recipients remained under anesthesia for the duration of extracorporeal support and recovery, is that we established a configuration in which swine recipients remained conscious throughout the procedure. Such an approach avoids the adverse effects of anesthetic agents and recipient immobility, provides access to nutrition ad libitum, and models a translational setup of XC between a patient and a donor organ. Throughout 4 days of normothermic support, all extracorporeal lungs and conscious swine recipients were subjected to longitudinal analyses to assess the safety and stability of the organ support system.

\section{METHODS}

\section{Study Design}

This study received approval from the Institutional Animal Care and Use Committee at Columbia University. In this proof-of-feasibility study, we investigated healthy swine lungs $(n=3)$ as a reproducible experimental input to assess the ability of the extracorporeal lung support system to maintain the structure, function, and integrity of extracorporeal lungs for 4 days, and to establish baseline values and methodologies. The mean total normothermic extracorporeal support time of all procedures was $100.7 \pm 1.2$ hours.

\section{Animals}

Six Yorkshire swine (3 donor-recipient pairs, aged 4-6 months) were used in this study. Swine lung donors had a mean weight of $43.3 \mathrm{~kg}$ (range, $35.7-57.0 \mathrm{~kg}$ ), and swine recipients had a mean weight of $53.0 \mathrm{~kg}$ (range, $41.5-59.0 \mathrm{~kg}$ ). No animals died in the course of this study.

\section{Donor Lung Procurement}

Swine lungs were procured in standard fashion as previously described. ${ }^{12,13}$ The mean duration of cold static lung preservation was $4.8 \pm 0.6$ hours.

\section{Extracorporeal Lung Cannulation}

A $20 \mathrm{~F}$ cannula was secured within the pulmonary artery, and the trachea was intubated with a $7.5 \mathrm{~mm}$ cuffed endotracheal tube, as previously described. ${ }^{12,13,17}$ The aortic arch, serving as an endothelialized biobridge between the lungs and the extracorporeal circuit, was secured to the left atrial cuff with a running $6-0$ polypropylene suture. A $36 \mathrm{~F}$ venous drainage 


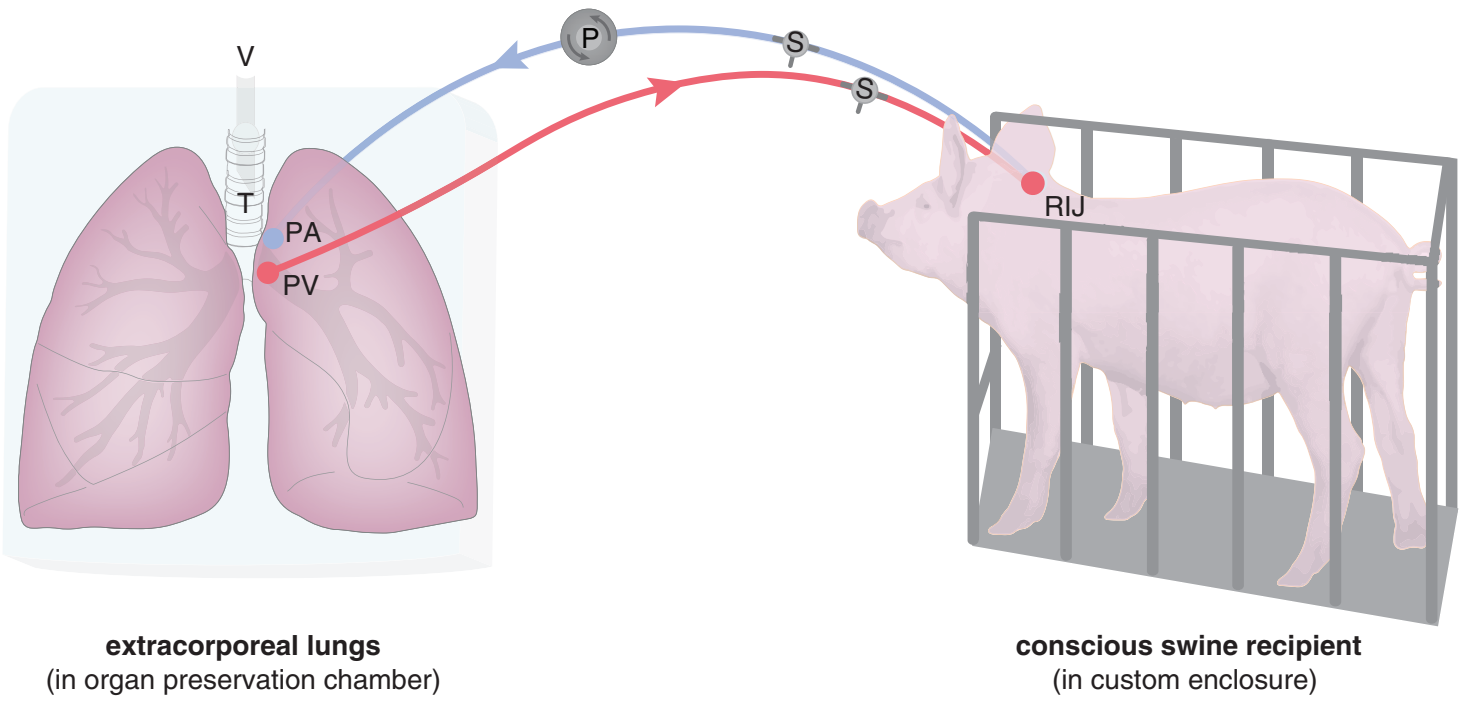

A (in organ preservation chamber)

(in custom enclosure)

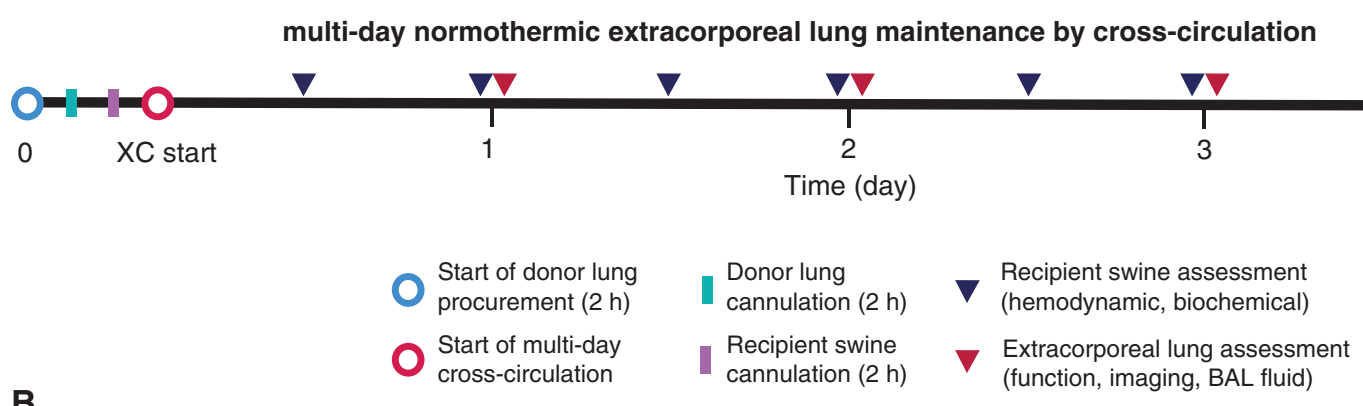

FIGURE 1. Experimental overview of multiday extracorporeal lung support system. A, Lungs were explanted from donor swine and the pulmonary artery $(P A)$ and pulmonary vein $(P V)$ were cannulated. Extracorporeal lungs were maintained in a humidified, normothermic preservation chamber and connected to a mechanical ventilator $(V)$ via endotracheal intubation $(T)$. Recipient swine were cannulated with a dual-lumen cannula via the right internal jugular $(R I J)$ vein. After recovery from anesthesia, recipient swine were placed in a custom enclosure for the duration of the procedure. The extracorporeal circuit contained a centrifugal pump $(P)$ that cross-circulated whole blood between the recipient swine and the extracorporeal lungs. In-line sensors $(S)$ monitored the hemodynamic stability of the recipient swine and extracorporeal lungs during multiday normothermic support. B, Experimental timeline. Extracorporeal lungs were maintained by normothermic cross-circulation $(X C)$ and periodically assessed for 4 days. $B A L$, Bronchoalveolar lavage.

cannula was secured to the biobridge with a 2-0 braided polyester tie. Lungs were placed in the organ preservation chamber in prone position in a sterile, double-lined organ basin containing warm normal saline.

\section{Recipient Swine Cannulation}

Recipient swine underwent general anesthesia after intramuscular induction with tiletamine $(5 \mathrm{mg} / \mathrm{kg})$. Cefazolin $(30 \mathrm{mg} / \mathrm{kg})$ and enrofloxacin $(5 \mathrm{mg} / \mathrm{kg})$ were administered before skin incision and re-dosed every 8 and 24 hours, respectively. Immunosuppression was administered intravenously: tacrolimus $(5 \mathrm{mg} / \mathrm{kg})$, mycophenolate mofetil $(500 \mathrm{mg})$, each redosed every 12 hours, and methylprednisolone $(125 \mathrm{mg})$, re-dosed every 8 hours. After exposing the right internal jugular vein, a heparin bolus $(15,000 \mathrm{U})$ was administered, and the vein was cannulated with a $19 \mathrm{~F}$ to 23F dual-lumen cannula (Avalon Elite; Maquet Cardiopulmonary, Rastatt, Germany) (Figure 2, $A$ and $B$ ).

\section{XC and Extracorporeal Lung Support}

Calcium gluconate $(1 \mathrm{~g})$ was administered intravenously to recipient swine, and cross-circulation of blood between recipient swine and extracorporeal lungs was initiated, as previously described. ${ }^{12,13}$ The extracorporeal circuit contained a pump console (Jostra HL-20; Maquet Cardiopulmonary), disposable pump (Rotaflow Centrifugal Pump; Maquet Cardiopulmonary), and continuous monitoring software (VIPER; Spectrum Medical, Cheltenham, England). Circuit flow rate was maintained within a protective regime between $5 \%$ and $10 \%$ of the estimated cardiac output of recipient swine, with pulmonary artery pressures $<20 \mathrm{~mm} \mathrm{Hg}$, and pulmonary vein pressures between 3 and $5 \mathrm{~mm} \mathrm{Hg}$ (Figure E1, A). ${ }^{12,13}$ Extracorporeal lungs were ventilated (Oxylog 3000 plus; Dräger, Lübeck, Germany) (Video 1) with the following settings: respiratory rate, 6 to $8 \mathrm{bpm}$; tidal volume, 6 to $8 \mathrm{~mL} / \mathrm{kg}$; positive endexpiratory pressure, $5 \mathrm{~cm} \mathrm{H}_{2} \mathrm{O}$; inspired oxygen fraction, $40 \%$; and maintained on $\mathrm{XC}$ for a mean duration of $1.5 \pm 0.1$ hours before initiating recovery of recipient swine from general anesthesia.

\section{Management of Recipient Swine During Recovery From Anesthesia}

Anesthetized recipient swine were transferred into a Panepinto sling suspended within a custom enclosure (Figure E2) and allowed to recover from anesthesia for a mean duration of $2.1 \pm 1.3$ hours (Figure 2, $C$ and 
Step 1: Swine venous cannulation (2 hours)

A

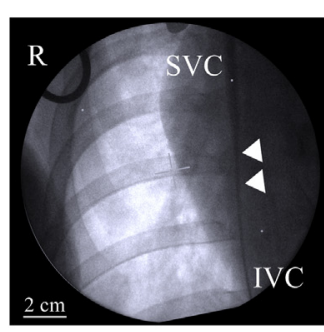

cannula placement

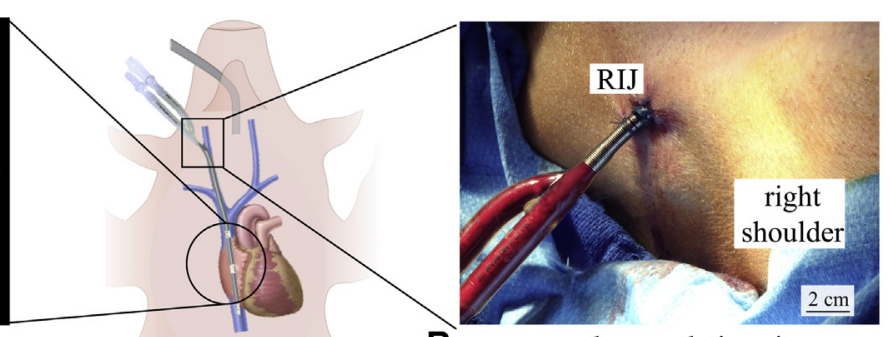

neck cannulation site

Step 2: Swine recovery from anesthesia in Panepinto sling (2 hours)

C
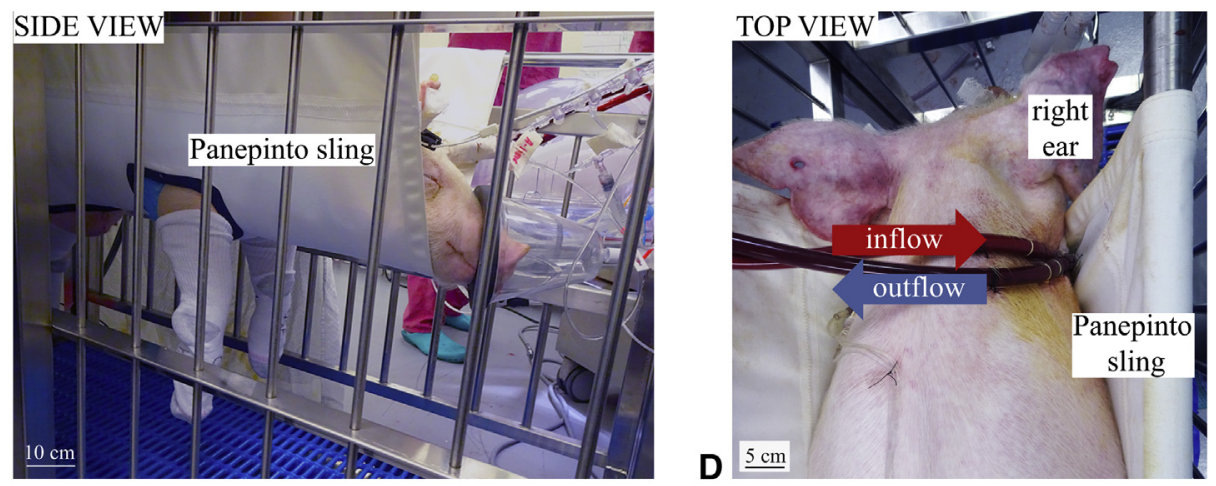

Step 3: Cross-circulation with conscious swine recipient in custom enclosure (4 days)

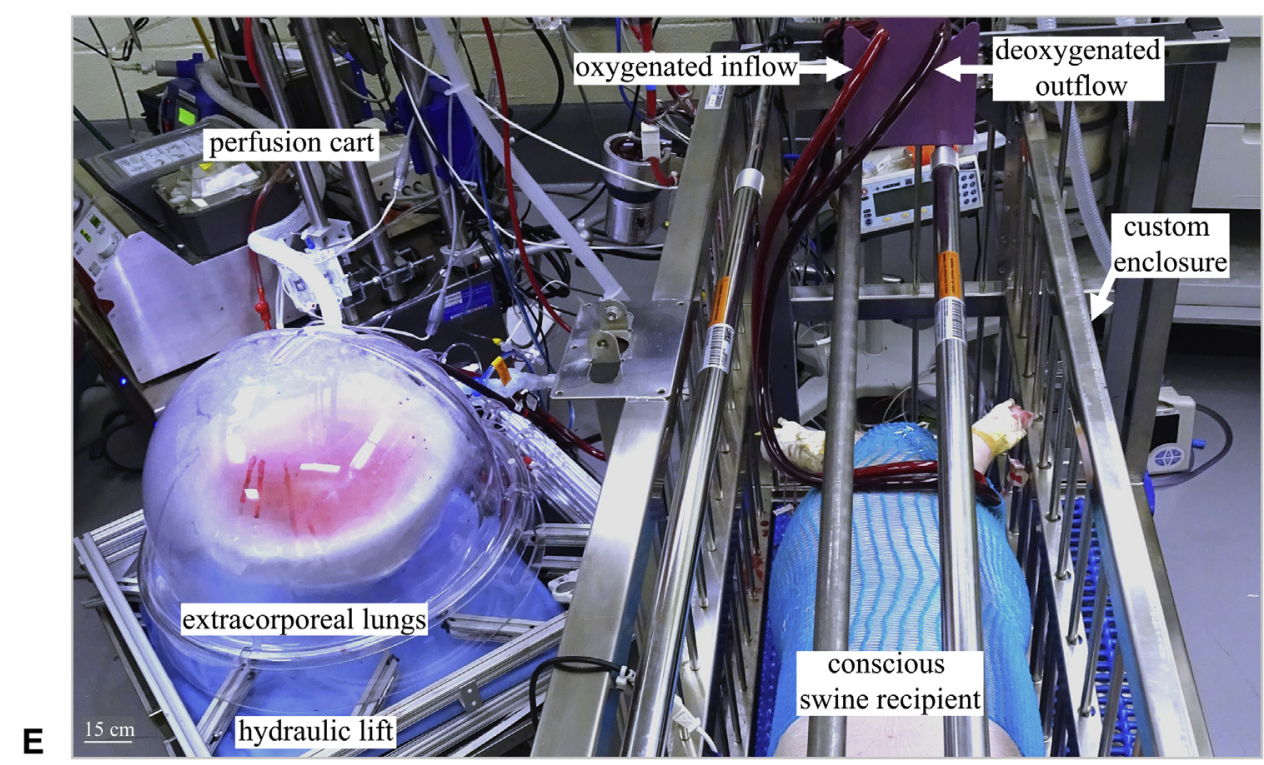

FIGURE 2. Experimental setup of multiday extracorporeal lung support system using a swine recipient. A, A dual-lumen cannula was placed in the right internal jugular $(R I J)$ vein under fluoroscopic guidance. White arrows indicate cannula. B, The cannula was tunneled along the lateral aspect of the right neck and secured to the dorsum of recipient swine. $\mathrm{C}$ and D, Following initiation of cross-circulation, cannulated recipient swine were transferred to a Panepinto sling where they remained elevated from the enclosure floor for safe recovery from anesthesia and extubation. E, Once conscious, recipient swine were lowered into a custom enclosure where they remained for the duration of the procedure. SVC, Superior vena cava; $R$, right; $I V C$, inferior vena cava.

$D)$. Following transfer to the Panepinto sling, recipient swine were weaned from general anesthesia and administered ketamine $(0.5-3.0 \mathrm{mg} / \mathrm{kg})$ and dexmedetomidine $(0.1-0.4 \mathrm{mg} / \mathrm{kg} / \mathrm{h})$ as needed. When spontaneous breathing was achieved, recipient swine were extubated while suspended in the Panepinto sling, and subsequently lowered onto the floor of the custom enclosure, where they were allowed to fully recover from 


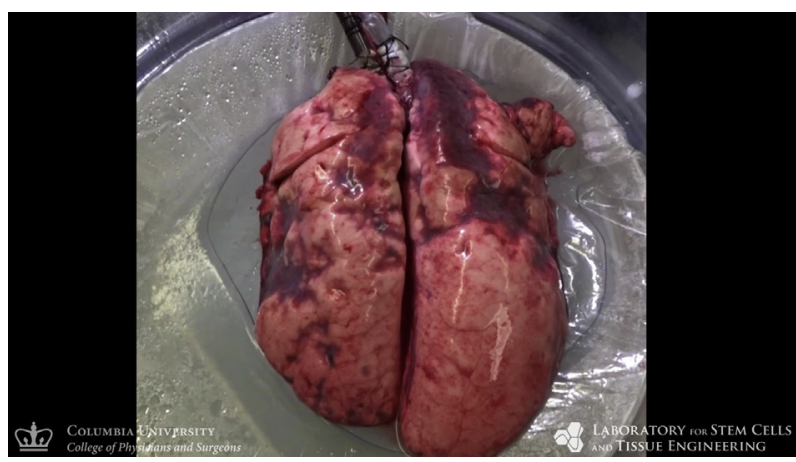

VIDEO 1. Normothermic maintenance of extracorporeal swine lungs with multiday lung support system. Video available at: https://www.jtcvs.org/ article/S0022-5223(19)32146-4/fulltext.

anesthesia (Video 2). Swine recipients were maintained in the custom enclosure for 4 days (Figure 2, E, and Video 3).

\section{Analyses of Extracorporeal Lungs and Recipient Swine}

Multiscale analyses of extracorporeal lungs were performed every 24 hours. Recipient swine were continuously monitored, and hemodynamic and biochemical parameters were recorded every 12 hours. Detailed methods are available in Appendix E1.

\section{RESULTS}

Extracorporeal lungs were connected to the normothermic support system and periodically evaluated over 4 days (Figure 1). Recipient swine were continuously monitored through behavioral observation, vital signs, and blood samples analyses.

\section{Extracorporeal Circuit Stability}

All extracorporeal circuit parameters were maintained within target lung-protective ranges throughout 4 days of normothermic support. Pulmonary artery pressures remained below $25 \mathrm{~mm} \mathrm{Hg}$ (Figure 3, A); and the transpulmonary pressure gradient, the difference between pulmonary artery and vein pressures, was maintained within the target

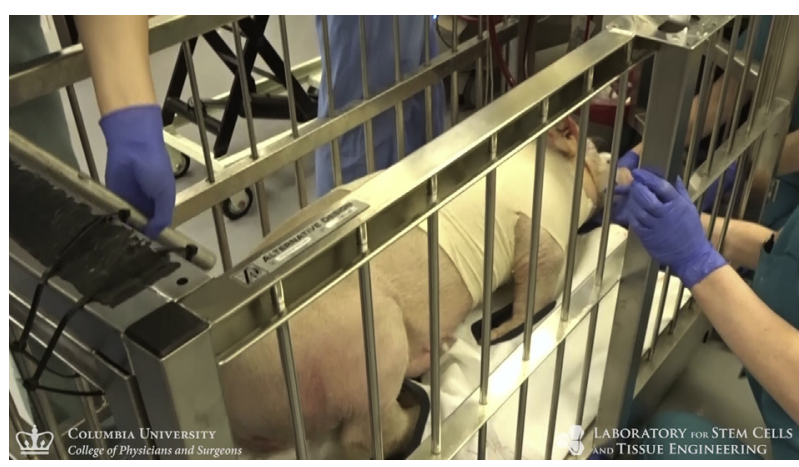

VIDEO 2. Transfer of swine recipient in Panepinto sling to custom enclosure. Video available at: https://www.jtcvs.org/article/S0022-5223(19) 32146-4/fulltext.

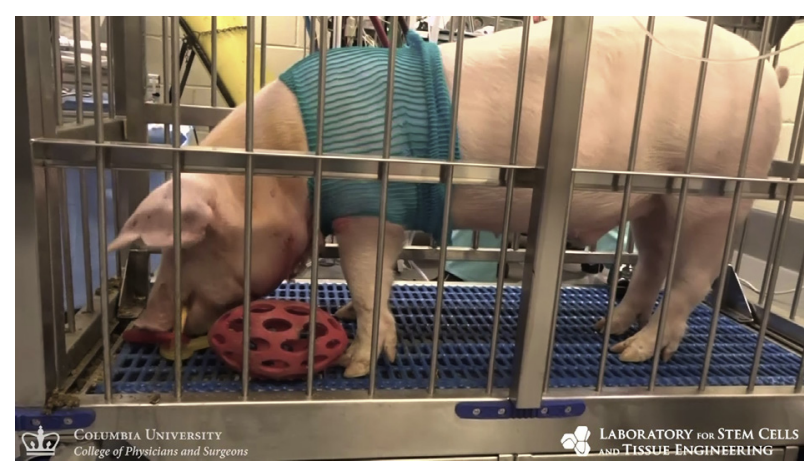

VIDEO 3. Active enrichment of recipient swine in custom enclosure throughout multiday lung support. Video available at: https://www.jtcvs. org/article/S0022-5223(19)32146-4/fulltext.

range of 5 to $15 \mathrm{~mm} \mathrm{Hg}$ (Figure 3,C). Flows were maintained on average at $0.28 \pm 0.03 \mathrm{~L} / \mathrm{min}(8 \%-9 \%$ of estimated cardiac output ${ }^{18}$ ) (Figure 3, B), and the circuit had a mean temperature of $35.1^{\circ} \mathrm{C} \pm 1.2^{\circ} \mathrm{C}$ throughout the 4 days (Figure $3, D$ ). The $\mathrm{pH}$ of the perfusate stayed within the physiologic range of $7.42 \pm 0.05$ (Figure 3,E), and lactate remained below $2 \mathrm{mmol} / \mathrm{L}$ until day 4 , when lactate increased slightly to $3.10 \pm 0.14 \mathrm{mmol} / \mathrm{L}$ (Figure $3, F$ ).

\section{Recipient Swine Safety and Stability}

All recipient swine tolerated venous neck cannulation (Figure 2, B), the experimental custom enclosure (Figure 2,E), and exhibited normal food and water consumption and excretion throughout all procedures. Safety and stability were assessed by monitoring of recipient swine hemodynamic parameters, which were maintained within normal ranges (mean heart rate, $112 \pm 18 \mathrm{bpm}$ and mean systolic pressure, $120 \pm 29 \mathrm{~mm} \mathrm{Hg}$ ), and by hemogas analysis (pH day 0, $7.36 \pm 0.02 ; \mathrm{pH}$ day 4, $7.37 \pm 0.08$ ) (Table E1). Hematocrit decreased gradually over 4 days of support (day $0,27.2 \% \pm 16.6 \%$; day $4,10.7 \% \pm 2.3 \%$ ) due to repeated blood sampling from both recipient swine and the extracorporeal circuit, and from minor transient bleeding from repeated lung tissue sampling. The inflammatory response of recipient swine was evaluated by quantification of serum inflammatory cytokine levels. From baseline to day 4, mean serum concentrations of granulocyte-macrophage colony-stimulating factor, interferon gamma (IFN $\gamma$ ), interleukin (IL) $1 \beta$, Il-1ra, IL-6, IL10 , and tumor necrosis factor alpha $(\mathrm{TNF} \alpha)$ variably increased, with the largest increases in IL-1 $\beta$ (10.4-fold), IFN $\gamma$ (19.2-fold), and IL-1ra (69-fold). Mean serum concentrations of IL- $1 \alpha$, IL-2, IL-4, IL-8, IL-12, and IL-18 decreased, with the largest decrease in IL-8 (14.8-fold) (Table E2). All serum cytokine concentrations after 4 days of normothermic support were within or below ranges reported in swine EVLP studies that provided a maximum of 12 hours of support (Table E3). 

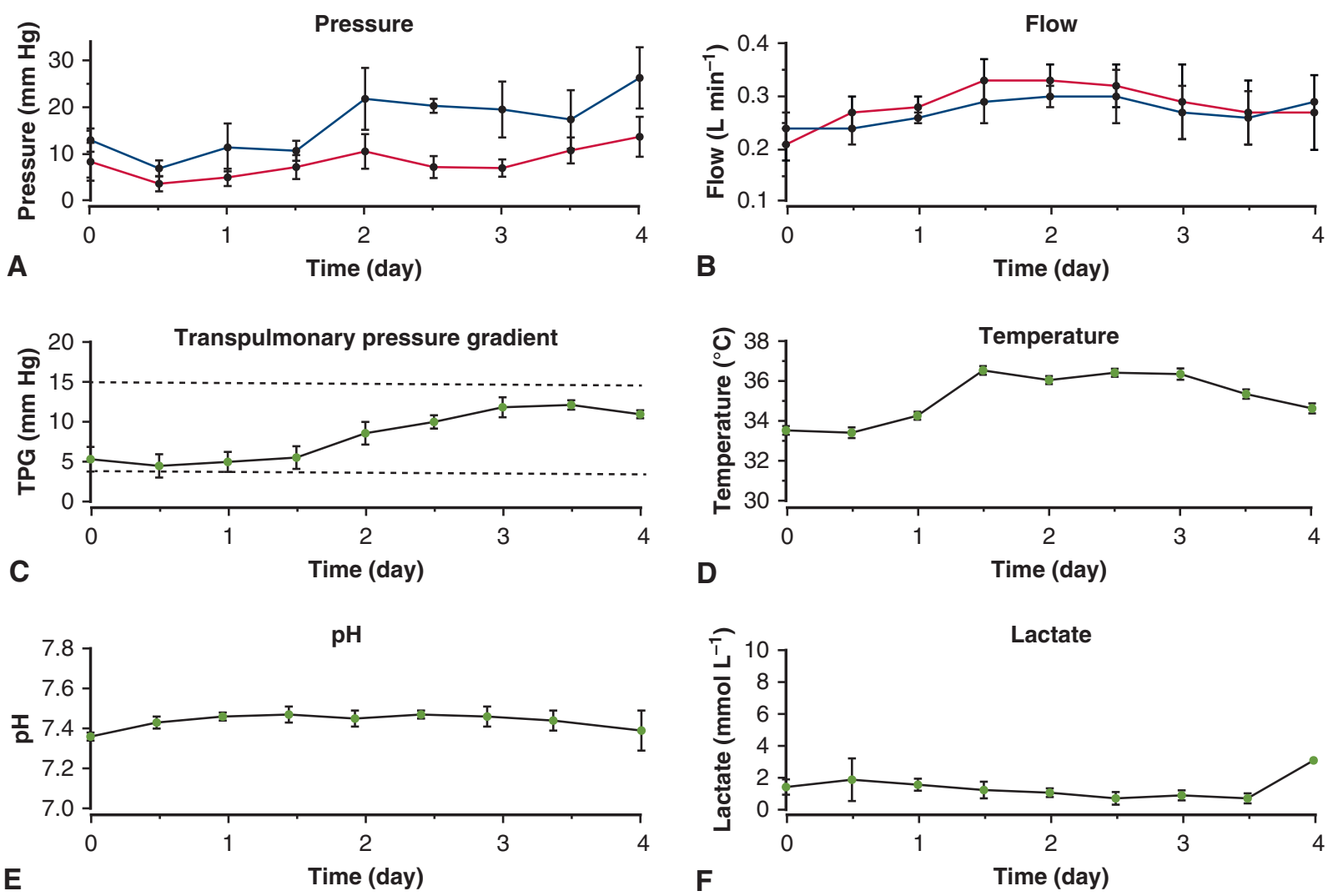

Pulmonary artery

Pulmonary vein

FIGURE 3. Stability of circuit parameters during multiday extracorporeal lung support. A, Pressure. B, Flow. C, Transpulmonary pressure gradient (TPG), which is the difference between pulmonary artery and pulmonary vein pressures. D, Temperature. E, pH. F, Lactate. Dotted lines define target range. Values are presented as mean \pm standard deviation.

\section{Functional Maintenance of Extracorporeal Lungs}

Respiratory function of extracorporeal lungs was preserved over 4 days of normothermic support. Robust gas exchange with mean arterial oxygen tension/inspired oxygen fraction values above $430 \mathrm{~mm} \mathrm{Hg}$ (day 0, $439.4 \pm 227.1 \mathrm{~mm} \mathrm{Hg}$; day 4, $548.5 \pm 176.9 \mathrm{~mm} \mathrm{Hg}$ ) (Figure 4, A), and dynamic compliance with mean values above $20 \mathrm{~mL} / \mathrm{cm} \mathrm{H}_{2} \mathrm{O}$ (day 0, $22.2 \pm 1.7 \mathrm{~mL} / \mathrm{cm} \mathrm{H}_{2} \mathrm{O}$; day 4, $20.0 \pm 1.0 \mathrm{~mL} / \mathrm{cm} \mathrm{H}_{2} \mathrm{O}$ ) (Figure $4, B$ ) were maintained consistently for the duration of all procedures. Mean peak inspiratory pressures increased slightly from day 0 to day 4 (day $0,20.5 \pm 0.7 \mathrm{~cm} \mathrm{H}_{2} \mathrm{O}$; day 4, $25.0 \pm 4.2 \mathrm{~cm} \mathrm{H}_{2} \mathrm{O}$ ) (Figure 4,C), but always remained below $30 \mathrm{~cm} \mathrm{H}_{2} \mathrm{O}$ for equivalent tidal volumes. All other functional parameters demonstrated minimal changes from baseline to day 4 (Table E4). Lung weight gradually increased over 4 days (day $0,0.84 \pm 0.17 \mathrm{~kg}$; day 4 , $1.18 \pm 0.07 \mathrm{~kg}$ ) (Figure 4,D), which was likely due to edema resulting from changes in hydrostatic pressure caused by variations in position of conscious swine recipients (eg, prone to standing) (Figure E1, B-D).

\section{Multiscale Analyses of Extracorporeal Lungs}

Gross photography of extracorporeal lungs showed normal appearance of the pleural surface with areas of localized consolidation periodically observed in upper lobes after day 2 (Figure 5 and Figure E3, $A$ ). Radiography confirmed that extracorporeal lungs remained aerated, with diffuse radiopacities in upper lobes after day 2 (Figure 5, C). Surface thermography revealed that lobes with lower surface temperatures were consistent with radiolucent areas (Figure 5, B), suggesting that consolidated regions in upper lobes had ventilation-perfusion mismatch with decreased ventilation leading to increased surface temperatures. ${ }^{19,20}$ Bronchoscopy confirmed normal appearance of large airways with no evidence of airway edema, erythema, or secretions after 4 days (Figure 5, D). Although histologic evaluations revealed edema in upper lobes, structural preservation of lung parenchyma, pulmonary airways, and the vascular tree was confirmed throughout the lungs (Figure 5, $E$ and $F$, and Figure E3, $B-D$ ). Transmission electron microscopy confirmed preservation of the blood-gas barrier with intact alveolar epithelial lining and abundant 

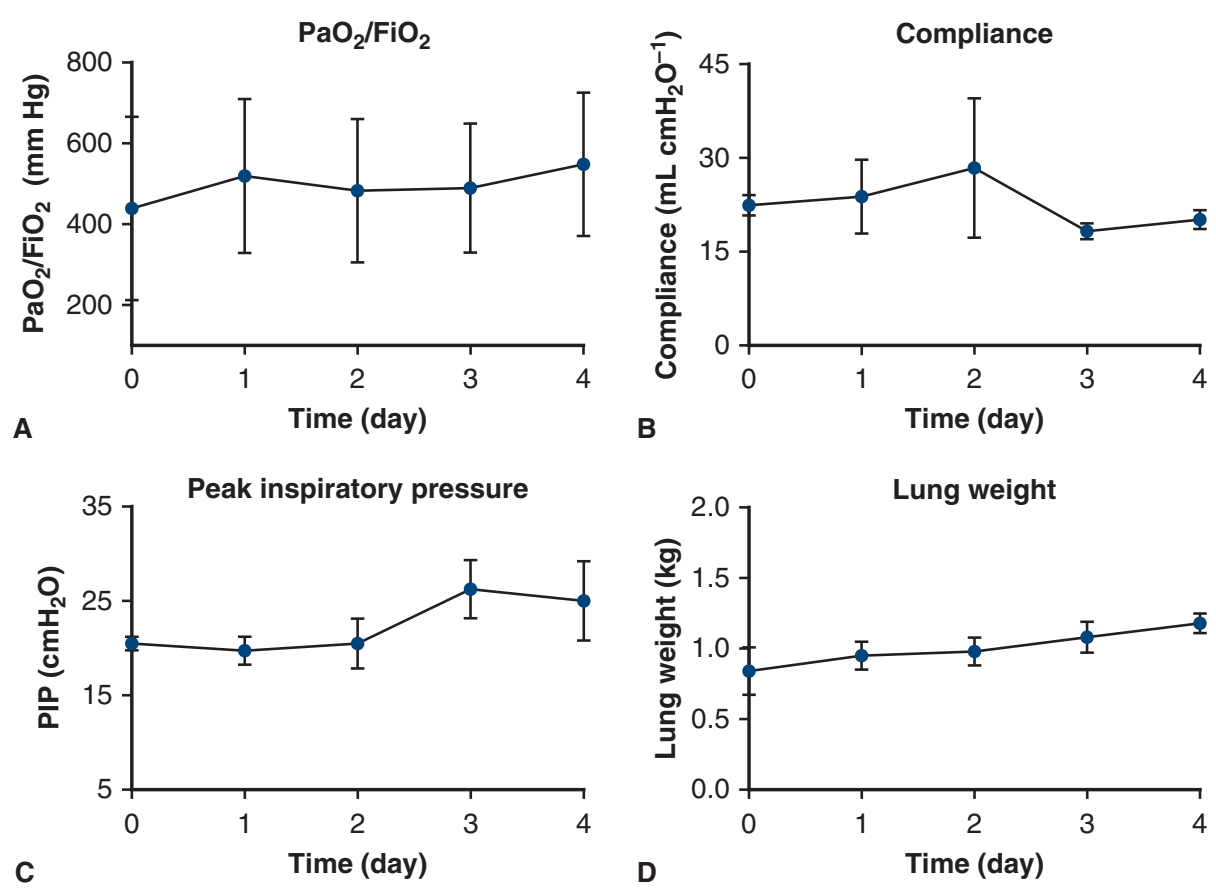

FIGURE 4. Maintenance of extracorporeal lung function for 4 days using multiday lung support system. A, Arterial oxygen tension/inspired oxygen fraction. B, Dynamic compliance. C, Peak inspiratory pressure $(P I P)$. D, Lung weight. Values are presented as mean \pm standard deviation.

type II pneumocytes with normal cuboidal morphology (Figure 5, G).

\section{Inflammation and Histopathologic Assessment of Extracorporeal Lungs}

Airway inflammation was assessed by quantification of inflammatory cytokines in bronchoalveolar lavage (BAL) fluid. From baseline to day 4, mean concentrations of granulocyte-macrophage colony-stimulating factor, IL-4, IL-6, and IL-10 trended upward but did not increase drastically, and mean concentrations of IFN $\gamma$, IL- $1 \alpha$, IL- $1 \beta$, IL1ra, IL-2, IL-8, IL-12, IL-18, and TNF $\alpha$ decreased in BAL fluid (Figure 6, $A-F$, and Table E5 and E6). Notably, the largest increase of inflammatory cytokine concentrations in BAL fluid was IL-4 (5.5-fold), and the largest decrease was IFN $\gamma$ (104.9-fold). To assess the degree of injury in extracorporeal lungs, tissue samples were subjected to blinded histopathologic review and assigned lung injury scores (Figure 6, $G$ ) according to an established injury scoring rubric (Figure E4, C). Polymorphonuclear cells, indicators of immune response to injury, remained low in airways but gradually increased in alveoli. Alveolar and interstitial edema increased slightly, which was consistent with the observed increase in lung weight. Nevertheless, the lack of significant increase in any lung injury category score from baseline to day 4 suggests that the extracorporeal lungs experienced minimal to no injury over 4 days of normothermic support.

\section{Cellular Integrity and Function of Extracorporeal Lungs}

Pentachrome staining confirmed preservation of bronchial structures, including airway mucosa, smooth muscle, cartilage (Figure 7, A), and bronchial epithelium with intact pseudostratified epithelium and airway cilia (Figure 7, B). In the respiratory zone, alveolar capillaries, and distal venules were well perfused (Figure 7,C), consistent with the outstanding respiratory performance at day 4 . Immunohistochemical staining for vascular endothelial cadherin enabled visualization of the intact endothelial lining of pulmonary vessels (Figure 7,E). Following administration of nebulized methacholine on day 4, extracorporeal lungs demonstrated rapid bronchoresponsiveness (Figure 7, $\mathrm{H}$ ) of airway smooth muscle (Figure 7,D). Cell viability in extracorporeal lungs was confirmed by uptake of viability marker carboxyfluorescein succinimidyl ester (Figure 7, $F$ ), and cellular metabolism in the parenchyma of extracorporeal lungs remained within range of the metabolic activity of lungs in vivo (Figure 7, $G$ ).

\section{DISCUSSION}

In this proof-of-feasibility study, we describe an extracorporeal organ support system capable of maintaining the structure, viability, and function of extracorporeal lungs for 4 days (Figure 8). This system extends the duration of normothermic extracorporeal lung support significantly beyond the capability of current ex vivo lung perfusion 
A

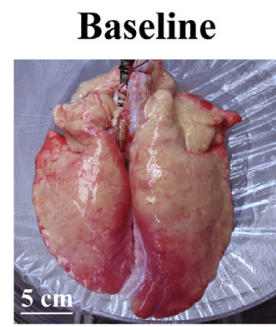

B

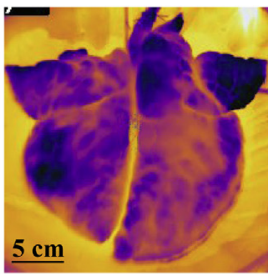

C

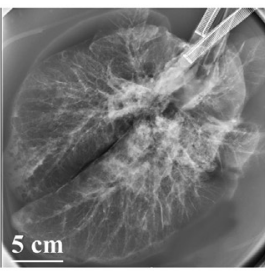

D

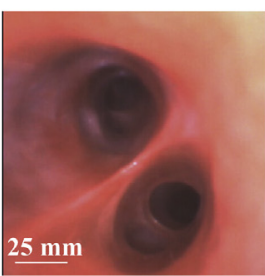

E

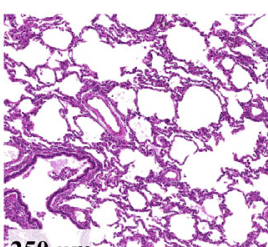

$250 \mu \mathrm{m}$
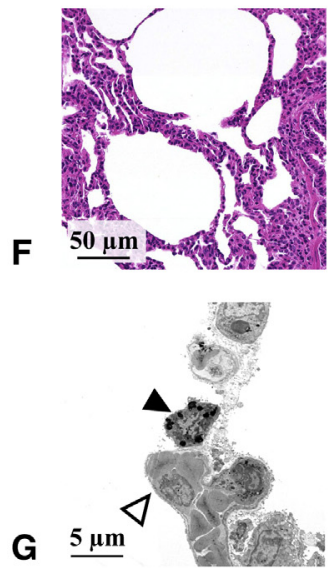

Day 1
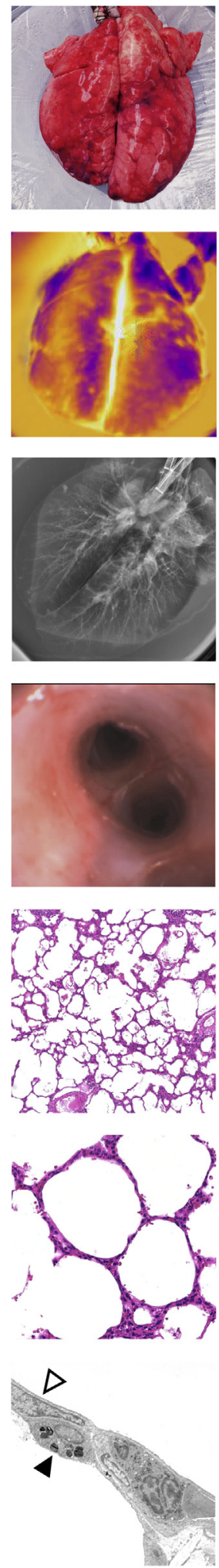

Day 2
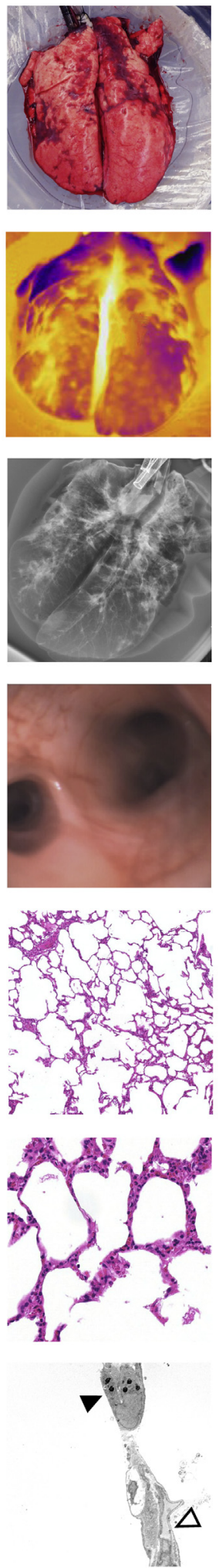

Day 3
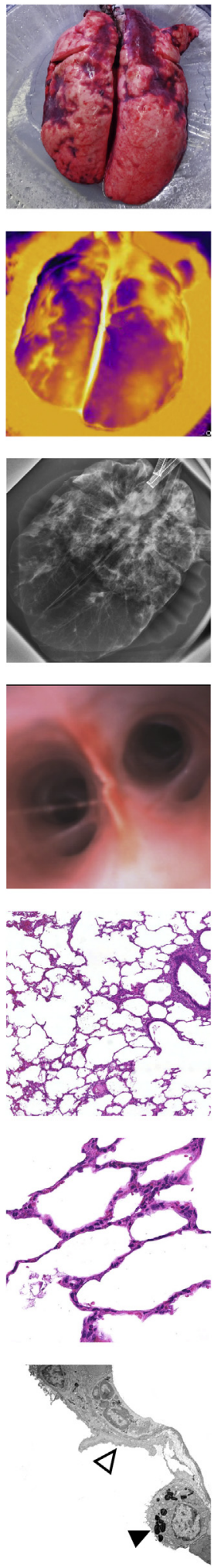

Day 4
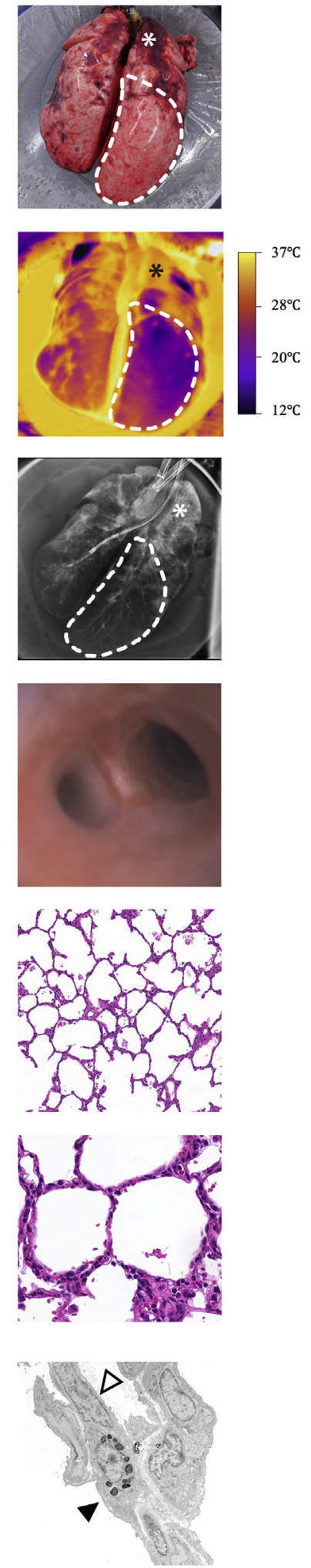

FIGURE 5. Multiscale analyses of extracorporeal lung maintenance and integrity. A, Photographic appearance. B, Thermographic appearance. C, Radiographic appearance. D, Bronchoscopic evaluation of extracorporeal lungs throughout 4 days of normothermic support, including aerated regions (dotted lines), and areas of local consolidation (stars). Microscopic analyses of bilateral lower lobes by hematoxylin and eosin staining. E, Low magnification. F, High magnification. G, Transmission electron microscopy revealed type II pneumocytes (black arrows) with visible lamellar bodies containing surfactant, and intact alveolar epithelial barrier (white arrows) of type I pneumocytes. 


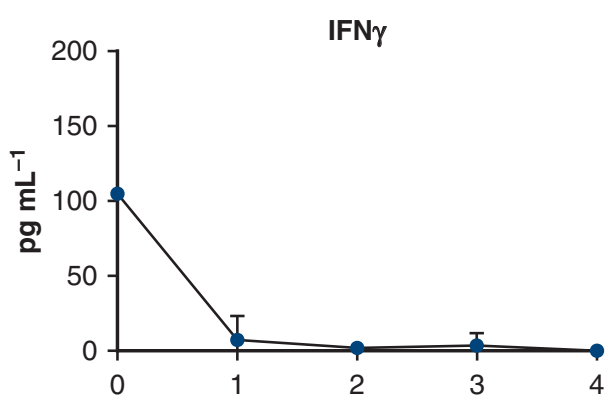

A

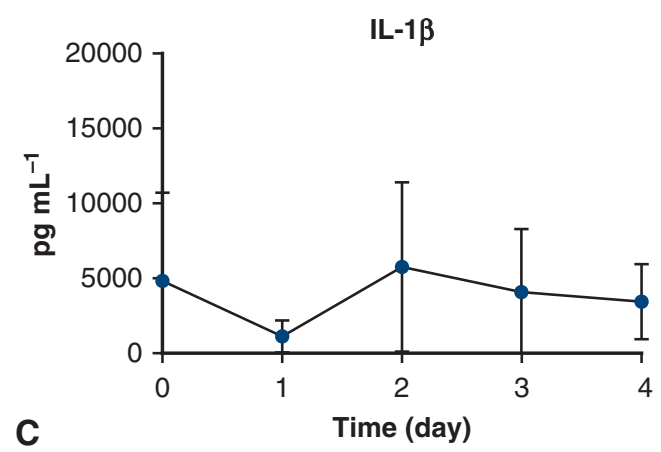

IL-8

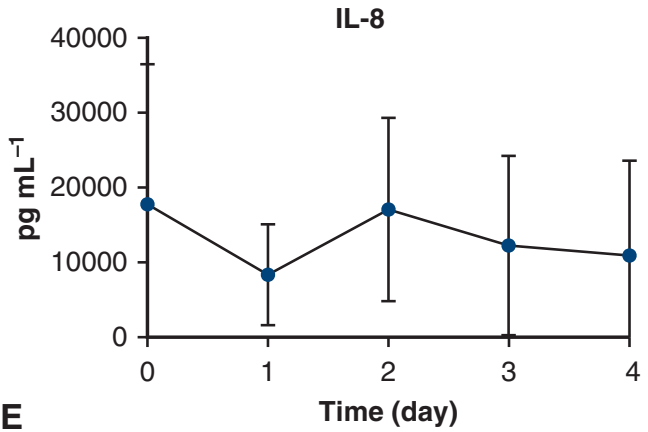

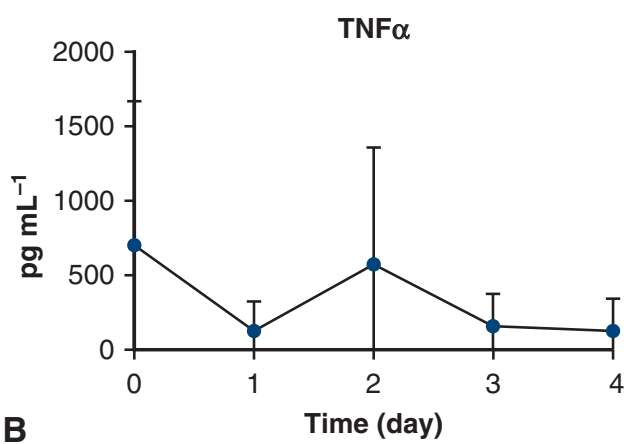

B

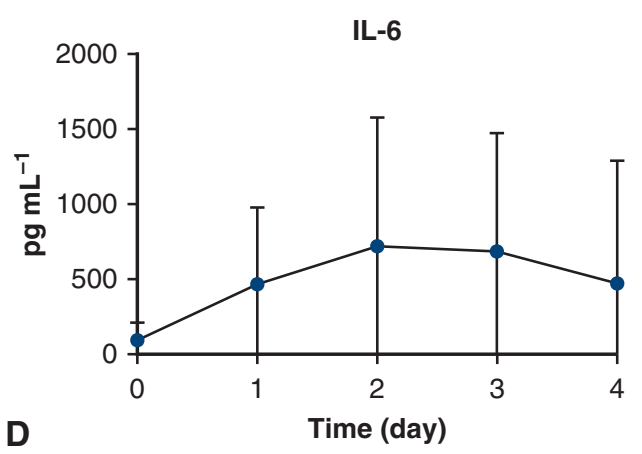

IL-10

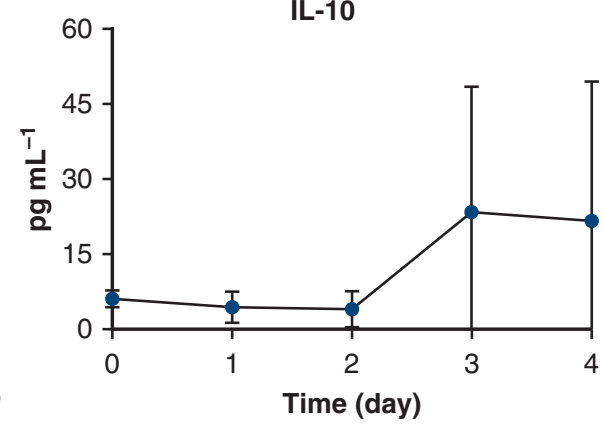

Lung injury scoring

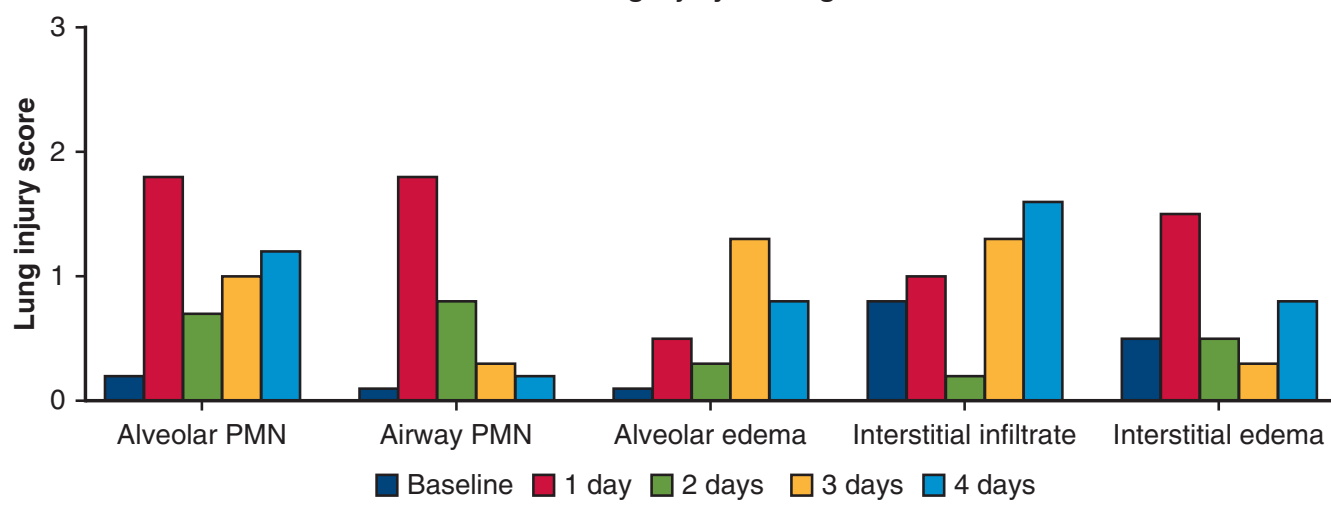

FIGURE 6. Quantification of airway cytokines and evaluation of lung injury during multiday extracorporeal lung support. Bronchoalveolar lavage fluid concentrations. A, Interferon gamma $(I F N \gamma)$. B, Tumor necrosis factor alpha $(T N F \alpha)$. C, Interleukin $(I L)$ 1 $\beta$. D, IL-6. E, IL-8. F, IL-10. G, Lung injury scoring by blinded histopathologic review. Values are presented as mean \pm standard deviation. PMN, Polymorphonuclear cells. 

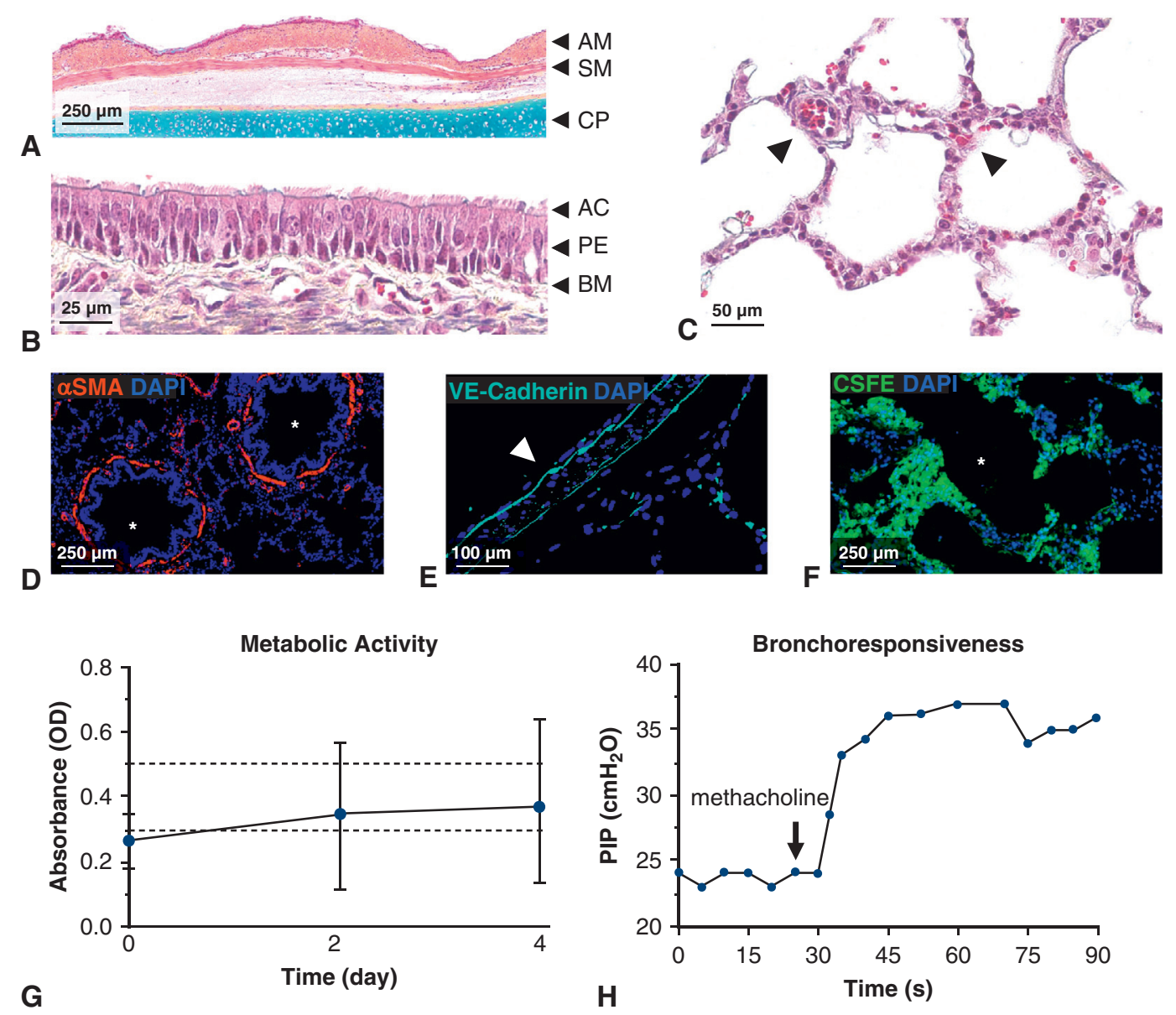

FIGURE 7. Cellular integrity and function in extracorporeal lungs after 4 days of normothermic support. Pentachrome staining. A, Large airways at low magnification with preserved airway mucosa $(A M)$, smooth muscle $(S M)$, and cartilage plate $(C P)$. B, Large airways at high magnification with outstanding preservation of airway cilia $(A C)$, pseudostratified epithelium $(P E)$, and basement membrane $(B M)$. C, Alveoli with intact blood-gas barrier and perfused venule and alveolar capillaries (arrows). D, Immunohistochemical staining was used to confirm retention of alpha smooth muscle actin $(\alpha S M A)$ around small airways (stars). E, Immunohistochemical staining was used to confirm retention of vascular endothelial (VE)-cadherin by endothelial cells in large and small vessels throughout the pulmonary vascular tree. F, Cell viability throughout the lung parenchyma was confirmed by pervasive uptake of carboxyfluorescein succinimidyl ester (CFSE). Star indicates alveolar space. G, Metabolic activity of lung parenchyma. Dotted lines indicate normal range of metabolic activity of healthy swine lungs in vivo. $\mathrm{H}$, Changes in peak inspiratory pressure after administration of nebulized methacholine (arrow). Values are presented as mean \pm standard deviation. PIP, Peak inspiratory pressure.

systems, from hours to multiple days. Our hypothesis is that homeostatic normothermic extracorporeal support for days to weeks could offer new opportunities for the assessment, recovery, and regeneration of donor lungs. Additionally, we established new methods to enable multiday $\mathrm{XC}$ of whole blood between extracorporeal lungs and a conscious large animal. Our results demonstrate feasibility that lungs can be maintained outside the body for 4 days with outstanding preservation of respiratory function, lung tissue structure, and cellular integrity and metabolism. Such a system offers a developmental platform for advanced therapeutic interventions such as gene or cell therapies for extracorporeal or intracorporeal organs.

Three new methodologies were developed in this study: a swine behavioral training program (Table E7 and
Figure E5) implemented 2 weeks before the start of XC to acclimate recipient swine to the custom enclosure used during the procedure, a technique to manage full recovery of anesthetized cannulated recipient swine to consciousness using a Panepinto sling and custom enclosure, and a novel extracorporeal circuit configuration with a single-site dual-lumen cannula to enable maintenance of circuit parameters within acceptable ranges. Altogether, these methods may prove useful in future translational studies involving the connection of conscious large animals or humans to extracorporeal circuits for organ support.

The function of extracorporeal lungs in this study was robustly maintained throughout 4 days of normothermic support. In comparison to previous studies, wherein swine lungs were supported by EVLP systems for a total of 


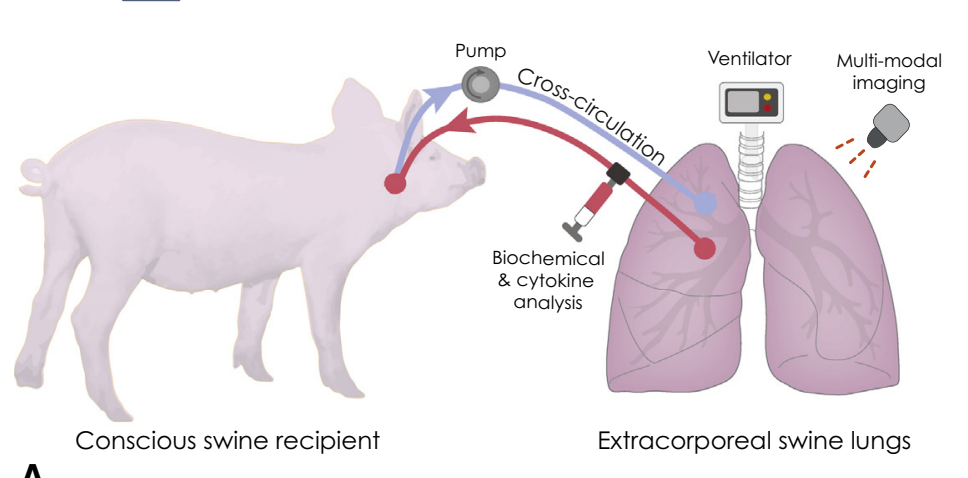

A

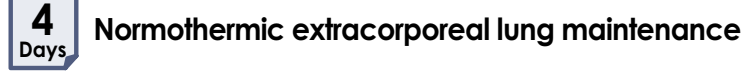

FIGURE 8. Multiday maintenance of extracorporeal lungs using cross-circulation with conscious swine: Experimental overview and results. A, Extracorporeal lungs were maintained using cross-circulation with conscious swine for a duration of 4 days. Lungs were placed in a normothermic organ preservation chamber and ventilated. Functional, biochemical, and multimodal imaging analyses were used to enable continuous monitoring of extracorporeal lungs and swine recipients. B, At day 4, lungs demonstrated maintenance of respiratory function, intact blood-gas barrier, cellular viability, and outstanding preservation of airway and alveolar architecture. PIP, Peak inspiratory pressure.

12 hours ${ }^{21}$ or 24 hours, ${ }^{22}$ lungs in this study at day 4 demonstrated superior mean arterial oxygen tension/inspired oxygen fraction (12 hours, $\sim 400 \mathrm{~mm} \mathrm{Hg} ; 24$ hours, $\sim 400 \mathrm{~mm}$ $\mathrm{Hg}$; XC 4 days, $548 \mathrm{~mm} \mathrm{Hg}$ ), equivalent mean compliance (12 hours, $20 \mathrm{~mL} / \mathrm{cm} \mathrm{H}_{2} \mathrm{O} ; 24$ hours, not reported; XC 4 days, $21 \mathrm{~mL} / \mathrm{cm}_{2} \mathrm{O}$ ), and in-range mean peak inspiratory pressures $\left(12\right.$ hours: $25 \mathrm{~cm} \mathrm{H}_{2} \mathrm{O} ; 24$ hours, $15 \mathrm{~cm} \mathrm{H}_{2} \mathrm{O}$; XC 4 days, $25 \mathrm{~cm} \mathrm{H}_{2} \mathrm{O}$ ). IL- $\beta$ and IL-8 in BAL fluid decreased from baseline to day 4 , and were only 1.4-fold and 2.2-fold higher, respectively, than in healthy swine lungs after 4 hours $^{23}$ of EVLP support. Furthermore, airway concentrations of IL-6, TNF $\alpha$, and IFN $\gamma$ after 4 days were, respectively, 2.2-fold, 2.8-fold, and 1260-fold lower. Altogether, these data suggest that the overall function and condition of swine lungs after 4 days were superior to the condition of lungs placed on 12 to 24 hours of EVLP support.

An established lung injury scoring rubric ${ }^{12,13}$ was used to assess the extent of lung injury. A comparison of lung injury scores after 4 days of support to scores previously reported in established swine lung models of ischemia reperfusion injury $^{12}$ and gastric aspiration injury ${ }^{13}$ revealed that lungs after 4 days showed less injury in all categories except for the mild interstitial edema reflected by increased lung weight. Overall, the composite injury score at day 4 in this study (4.6) was markedly lower than composite injury scores reported for ischemia reperfusion injury (6.8) and gastric aspiration (9.3) studies. When compared with recipient swine lungs at the conclusion of the procedure, extracorporeal lungs showed only a minimal increase in mean injury score (day 4, 4.6; recipient lungs, 3.8) (Figure E6).

The maintenance of vascular pressures within physiological range is critical for the prevention of pulmonary edema and the preservation of extracorporeal lungs. ${ }^{24}$ In this study, the pressure at the pulmonary veins was dependent on the hydrostatic pressure difference between the extracorporeal lungs and recipient swine, and was regulated by adjusting the height difference between the lungs and recipient swine (target, $10 \mathrm{~cm}$ ). In our previous studies, this height difference was fixed, as recipient swine were anesthetized and therefore immobile for the duration of all procedures. In this study, recipient swine were conscious and free to stand upright or lay prone at will, which resulted in intermittent changes in the height difference between the extracorporeal lungs and recipient swine. Adjustments to the height of the lungs were therefore necessary to maintain the target height difference of $10 \mathrm{~cm}$. Controlled adjustments of lung height were performed manually using a hydraulic lift, but were technically challenging to perform in real time. Consequently, variabilities in pulmonary vein pressures resulting from changes in swine position, in conjunction with the persistent prone position of extracorporeal lungs, likely contributed to the development of dependent interstitial edema, most notably observed in the upper lobes.

\section{Limitations}

There are several limitations to the present study. This study involved a small number of procedures $(n=3)$. Although such a study size limits the opportunity for statistical analyses, the results demonstrated feasibility of normothermic support of extracorporeal lungs for 4 days. Future studies will investigate larger numbers of lungs, recipient swine, and a wider variety of experimental conditions including increased flow rates, recovery of damaged lungs, and therapeutic interventions. Further, because immunosuppression was used, immunological markers of 
injury may be different than in the absence of immunosuppression, which may account for differences observed between cytokine concentrations in BAL fluid and serum. Notably, several of the cytokines investigated in this study have been shown to have both pro- and anti-inflammatory roles, ${ }^{25-27}$ so their functions in this system are difficult to interpret. Future studies using a multiday extracorporeal support system could help elucidate the roles of cytokines in extracorporeal lung support systems. Several technical challenges remain to be resolved in the current system: specification and regulation of a long-term extracorporeal organ environment, controlled variability of extracorporeal organ orientation, and appropriate ventilation and perfusion management strategies. The inability to strictly regulate dynamic hydrostatic pressure changes led to the development of edema, necessitating future development of feedbackregulated pump controls and an automated organ height adjustment system capable of responding precisely in real time to dynamic changes in swine position, transpulmonary pressure gradient, and lung weight. Although this study did not investigate deposition of recipient cells or platelets in extracorporeal lungs, which could result in plateletinduced injury and chimerism, future studies of hematologic and immunologic interactions will be critical to assess the safety of clinical translation.

Despite these limitations, this study demonstrated that cross-circulation enables a quality and duration of extracorporeal lung support not previously shown by EVLP systems. The use of XC to recover damaged lungs ex situ could be applicable in clinical settings where patients receiving extracorporeal membrane oxygenation fail to match suitable donor lungs. In such patients, XC of lungs with reversible injuries for several days could enable functional assessment, lung-protective strategies, and graft recovery while avoiding the physiologic insult associated with major surgical intervention and severe primary graft dysfunction. Lungs recovered by XC would then be transplanted into the patient, thereby potentially decreasing the morbidity and mortality associated with transplantation of injured lungs. Future investigations using multiday extracorporeal organ support could also enable advanced interventions through immunomodulation, ${ }^{28,29}$ cell replacement, ${ }^{17,30-33}$ or other bioengineering approaches, ${ }^{17,32,34-36}$ and ultimately serve as a platform to improve transplant outcomes.

\section{CONCLUSIONS}

In this study we demonstrate the longest duration of normothermic support of extracorporeal lungs reported to date (4 days), with outstanding maintenance of lung tissue and respiratory functions. We envision that this system could be applicable in clinical settings to recover and regenerate damaged donor organs, and in translational research settings as a platform to investigate new strategies for lung bioengineering.

\section{Webcast}

You can watch a Webcast of this AATS meeting presentation by going to: https://aats.blob.core.windows.net/ media/19\%20AM/Saturday_May4/203AC/203AC/S24\% 20-\%20Mechanical\%20Lung\%20Support/S24_3_webcast_ 120637225.mp4.

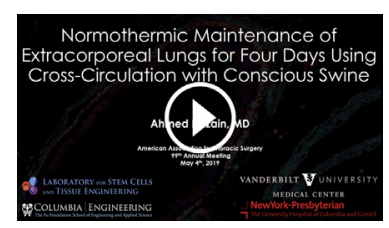

\section{Conflict of Interest Statement}

Drs Guenthart, O'Neill, Vunjak-Novakovic, Bacchetta, and Mr Fung have a pending patent for a cross-circulation platform for recovery, regeneration, and maintenance of extracorporeal organs. All other authors have nothing to disclose with regard to commercial support.

The authors thank the Institute of Comparative Medicine veterinary staff, including A. Romanov, S. Robertson, R. Ober, A. McLuckie, G. Geist, N. Herndon, S. Hastings, D. Ordanes, and A. Rivas for supporting animal studies; Weill Cornell Microscopy and Image Analysis Core Facility staff, including L. Cohen-Gould and J. P. Jimenez for transmission electron microscopy imaging services. The authors also thank the Herbert Irving Comprehensive Cancer Center Molecular Pathology Shared Resources, including T. Wu, D. Sun, and R. Chen for histology services; E. Lopes, G. Pierre, and I. Fedoriv for support with technical analytics; and S. Pistilli and S. Halligan for administrative support.

\section{References}

1. Ware LB, Wang Y, Fang X, Warnock M, Sakuma T, Hall TS, et al. Assessment of lungs rejected for transplantation and implications for donor selection. Lancet. 2002;360:619-20.

2. Singer J, Chen J, Blanc PD, Leard LE, Kukreja J, Chen H. A thematic analysis of quality of life in lung transplant: the existing evidence and implications for future directions. Am J Transplant. 2013;13:839-50.

3. Kugler C, Gottlieb J, Warnecke G, Schwarz A, Weissenborn K, Barg-Hock H, et al. Health-related quality of life after solid organ transplantation: a prospective, multiorgan cohort study. Transplant J. 2013;96:316-23.

4. Pinezich M, Vunjak-Novakovic G. Bioengineering approaches to organ preservation ex vivo. Exp Biol Med. 2019;244:630-45.

5. Guibert EE, Petrenko AY, Balaban CL, Somov AY, Rodriguez JV, Fuller BJ. Organ preservation: current concepts and new strategies for the next decade. Transfus Med Hemotherapy. 2011;38:125-42.

6. Makdisi G, Makdisi T, Jarmi T, Caldeira CC. Ex vivo lung perfusion review of a revolutionary technology. Ann Transl Med. 2017;5:343.

7. Tane S, Noda K, Shigemura N. Ex vivo lung perfusion: a key tool for translational science in the lungs. Chest. 2017;151:1220-8.

8. Steen S, Sjöberg T, Pierre L, Liao Q, Eriksson L, Algotsson L. Transplantation of lungs from a non-heart-beating donor. Lancet. 2001;357:825-9.

9. Cypel M, Yeung JC, Liu M, Anraku M, Chen F, Karolak W, et al. Normothermic ex vivo lung perfusion in clinical lung transplantation. N Engl J Med. 2011;364: 1431-40. 
10. Warnecke G, Van Raemdonck D, Smith MA, Massard G, Kukreja J, Rea F, et al. Normothermic ex-vivo preservation with the portable Organ Care System Lung device for bilateral lung transplantation (INSPIRE): a randomised, open-label, non-inferiority, phase 3 study. Lancet Respir Med. 2018;6:357-67.

11. Cypel M, Yeung JC, Machuca T, Chen M, Singer LG, Yasufuku K, et al. Experience with the first 50 ex vivo lung perfusions in clinical transplantation. $J$ Thorac Cardiovasc Surg. 2012;144:1200-7.

12. O'Neill JD, Guenthart BA, Kim J, Chicotka S, Queen D, Fung K, et al. Cross-circulation for extracorporeal support and recovery of the lung. Nat Biomed Eng. 2017:1:0037.

13. Guenthart BA, O'Neill JD, Kim J, Queen D, Chicotka S, Fung K, et al. Regeneration of severely damaged lungs using an interventional cross-circulation platform. Nat Commun. 2019;10:1985.

14. Van Raemdonck D, Hartwig MG, Hertz MI, Davis RD, Cypel M, Hayes D Jr, et al. Report of the ISHLT working group on primary lung graft dysfunction part IV: prevention and treatment: a 2016 consensus group statement of the International Society for Heart and Lung Transplantation. J Heart Lung Transplant. 2017;36:1121-36.

15. Song JH, Park JE, Lee JG, Lee CY, Nam KS, Suh JW, et al. Outcomes of perioperative extracorporeal membrane oxygenation use in patients undergoing lung transplantation. J Thorac Dis. 2017;9:5075-84.

16. Hoetzenecker K, Schwarz S, Muckenhuber M, Benazzo A, Frommlet F, Schweiger $\mathrm{T}$, et al. Intraoperative extracorporeal membrane oxygenation and the possibility of postoperative prolongation improve survival in bilateral lung transplantation. J Thorac Cardiovasc Surg. 2018;155:2193-206.e3.

17. Guenthart BA, O’Neill JD, Kim J, Fung K, Vunjak-Novakovic G, Bacchetta M. Cell replacement in human lung bioengineering. J Heart Lung Transplant. 2019; 38:215-24.

18. Lin H-Y, Freed D, Lee TW, Arora RC, Ali A, Almoustadi W, et al. Quantitative assessment of cardiac output and left ventricular function by noninvasive phasecontrast and cine MRI: validation study with invasive pressure-volume loop analysis in a swine model. J Magn Reson Imaging. 2011;34:203-10.

19. West JB. How well designed is the human lung? Am J Respir Crit Care Med. 2006;173:583-4.

20. Motoyama H, Chen F, Hijiya K, Kondo T, Ohata K, Takahashi M, et al. Novel thermographic detection of regional malperfusion caused by a thrombosis during ex vivo lung perfusion. Interact Cardiovasc Thorac Surg. 2015;20:242-7.

21. Aboelnazar NS, Himmat S, Hatami S, White CW, Burhani MS, Dromparis P, et al. Negative pressure ventilation decreases inflammation and lung edema during normothermic ex-vivo lung perfusion. J Heart Lung Transplant. 2018;37: 520-30.

22. Sommer W, Salman J, Avsar M, Hoeffler K, Jansson K, Siemeni TN, et al. Prediction of transplant outcome after 24-hour ex vivo lung perfusion using the organ care system in a porcine lung transplantation model. Am J Transplant. 2019; 19:345-55.

23. Roman M, Gjorgjimajkoska O, Neil D, Nair S, Colah S, Parmar J, et al. Comparison between cellular and acellular perfusates for ex vivo lung perfusion in a porcine model. J Heart Lung Transplant. 2015;34:978-87.

24. Linacre V, Cypel M, Machuca T, Nakajima D, Hashimoto K, Zamel R, et al. Importance of left atrial pressure during ex vivo lung perfusion. J Heart Lung Transplant. 2016;35:808-14.

25. Scheller J, Chalaris A, Schmidt-Arras D, Rose-John S. The pro- and antiinflammatory properties of the cytokine interleukin-6. Biochim Biophys Acta. 2011;1813:878-88

26. Arend WP, Malyak M, Guthridge CJ, Gabay C. Interleukin-1 receptor antagonist: role in biology. Annu Rev Immunol. 1998;16:27-55.

27. Cavaillon JM. Pro- versus anti-inflammatory cytokines: myth or reality. Cell Mol Biol. 2001;47:695-702.

28. Cypel M, Liu M, Rubacha M, Yeung JC, Hirayama S, Anraku M, et al. Functional repair of human donor lungs by IL-10 gene therapy. Sci Transl Med. 2009;1:4ra9.

29. Mordant P, Nakajima D, Kalaf R, Iskender I, Maahs L, Behrens P, et al. Mesenchymal stem cell treatment is associated with decreased perfusate concentration of interleukin-8 during ex vivo perfusion of donor lungs after 18-hour preservation. J Heart Lung Transplant. 2016;35:1245-54.

30. Gennai S, Monsel A, Hao Q, Park J, Matthay MA, Lee JW. Microvesicles derived from human mesenchymal stem cells restore alveolar fluid clearance in human lungs rejected for transplantation. Am J Transplant. 2015;15:2404-12.

31. La Francesca S, Ting AE, Sakamoto J, Rhudy J, Bonenfant NR, Borg ZD, et al. Multipotent adult progenitor cells decrease cold ischemic injury in ex vivo perfused human lungs: an initial pilot and feasibility study. Transplant Res. 2014:3:19.
32. Pinezich M, Vunjak-Novakovic G. Bioengineering approaches to organ preservation ex vivo. Exp Biol Med. 2019;244:630-45.

33. Mao AS, Mooney DJ. Regenerative medicine: current therapies and future directions. Proc Natl Acad Sci U S A. 2015;112:14452-9.

34. Dorrello NV, Guenthart BA, O’Neill JD, Kim J, Cunningham K, Chen YW, et al Functional vascularized lung grafts for lung bioengineering. Sci Adv. 2017;3: e1700521.

35. Gilpin SE, Charest JM, Ren X, Ott HC. Bioengineering lungs for transplantation. Thorac Surg Clin. 2016;26:163-71.

36. Ott HC, Clippinger B, Conrad C, Schuetz C, Pomerantseva I, Ikonomou L, et al Regeneration and orthotopic transplantation of a bioartificial lung. Nat Med. 2010;16:927-33.

Key Words: acute lung injury, airway lavage, alveolar recruitment, bronchoalveolar lavage fluid, chimerism, cross-circulation, extracorporeal membrane oxygenation, ex vivo lung perfusion, infrared thermography, lung bioengineering, lung transplantation, medical thermography, normothermic organ perfusion, organ shortage, regenerative medicine, swine model, tissue engineering, transplantation, whole organ bioreactor

\section{Discussion}

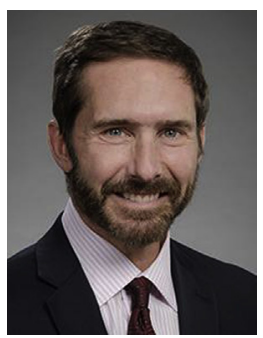

Dr Michael S. Mulligan (Seattle, Wash). This is a lot of work and it is very provocative. I have a couple of questions and I will ask them one at a time. Current iterations of ex vivo lung perfusion (EVLP) platforms are limited by several factors: they don't clear metabolic waste, they dilute them; they don't account for generated biologics; they don't have a plethora of homeostatic mechanisms that are active or meaningful; they ventilate and perfuse the nonphysiologic perimeters; and they maintain the static position that we know is a point of vulnerability for the development of dependent edema.

Based on what you have learned and what you have seen, how would you prioritize and what you offer as suggestions for revisions of current EVLP platforms to make them better and safer, particularly for extended perfusion times?

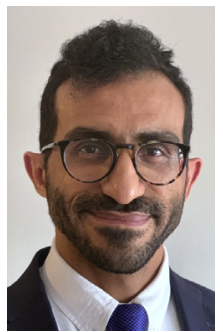

Dr Ahmed E. Hozain (New York, NY). Thank you Dr Mulligan for that question. When it comes to EVLP versus cross-circulation, I think there is room for both platforms. The platforms are different and serve different purposes. EVLP has been shown to recover marginal lungs and may facilitate treatment of hepatitis $\mathrm{C}$-infected lungs.

Our cross-circulation system is aimed at extending homeostatic physiologic support to enable regeneration and advanced bioengineering techniques that were not possible 
on EVLP due to its limited duration of support as it lacks several features that you mentioned, such as metabolic clearance and providing critical factors required for cellular regeneration. We want to use this platform to not only recover severely injured lungs but also to investigate possible new therapeutics that we wouldn't be able to do in vivo.

Dr Mulligan. Following up on that, I got the sense in reading your manuscript that maybe at the 4-day mark you are bumping into your wall. You started to see more edema, more alveolar infiltrates; you saw extension of the sort of subpleural thickening. Do you think the revisions that you can make now can further extend beyond 4 days, because they are allowing pneumonia to recover, traumatic injury to recover. You may need more time. Do you have practical steps for your next iteration?

Dr Hozain. This was a feasibility study to extend the duration of support to 4 days and we learned several things along the way, especially technical issues. Variability in static pressure in the pulmonary veins of the donor lung occurred as the animal would stand or lie down and required recurrent height adjustments to maintain a constant static pressure. We are working on a system that would allow for automatic adjustment to better maintain stability in pressure gradients. Along these lines and as you suggested earlier, the organ cannot lie in a static position for an extended period of time without suffering from dependent edema. As we extended the duration of support to 4 days, we realized that the lungs would develop dependent edema. We have constructed a solution to this problem and have started testing it. The extended duration platform has forced us to solve new problems that do not manifest in static positioning for 24- or 36-hour runs. We are confronted with developing mechanisms to maintain the external environment of the extracorporeal organ.

Dr Mulligan. My second question relates to other organ systems. How well do you think this technology translates to other organ systems and what experience do you folks have with other organs?

Dr Hozain. Our platform is adaptable to other organ systems, and we hope to present that work in the near future. 


\section{APPENDIX E1. DETAILS OF PREPROCEDURE AND PROCEDURE METHODS \\ Preprocedure Behavior Training of Recipient Swine}

Before initiation of cross-circulation, a swine behavior training program (Table E7) was implemented to acclimate recipient swine to the custom enclosure (Figure E2). Target training (ie, conditioning swine to touch nose to target when the trainer activated an audible clicker) was initiated at least 14 days before the start of cross-circulation and was used to encourage recipient swine to enter the custom enclosure by day 4 of training (Figure E5, A). Initially, swine were maintained in the custom enclosure for up to 1 hour. Through days 5 to 14 of training, recipient swine were maintained in the custom enclosure for at least 2 hours per day to increase comfort and familiarity with the experimental environment. The width of the custom enclosure was incrementally reduced to limit rotational movements (Figure E5, B). Behavior training was essential to minimize stress and maximize comfort of recipient swine, and thus decrease risk of decannulation during cross-circulation procedures. Throughout the preprocedure behavior training program, recipient swine experienced enrichment with manipulata (Figure E5, C), social interactions with procedure personnel (Figure E5, D), and standard and high-quality edible treats.

\section{Management and Monitoring of Recipient Swine}

During cannulation of the right internal jugular vein, physiologic parameters of anesthetized recipient swine, including heart rate, electrocardiogram, blood pressure (cuff and arterial line), oxygen saturation, end-tidal carbon dioxide, temperature, and respiratory rate were continuously monitored and recorded. Following recovery from anesthesia and throughout the duration of procedures, recipient swine were continuously monitored. Physical examinations to monitor and record respiratory signs (ie, dyspnea, tachypnea, coughing, and hypoxia), change in appetite, abnormal attitude or mentation, and signs of pain or discomfort were conducted by a large-animal veterinarian and animal behavior specialist at least twice daily. Throughout the duration of cross-circulation, recipient swine were maintained on a continuous intravenous heparin infusion (initial rate, $25 \mathrm{U} / \mathrm{kg} / \mathrm{h}$ ). Activated clotting time was measured and recorded hourly, and heparin infusion rates were titrated accordingly to remain within the target activated clotting time range of 200 to $300 \mathrm{sec}-$ onds. Recipient swine were allowed to eat, drink, sleep, and play freely in the custom enclosure. Twice daily, recipient swine received food (Laboratory Mini-Pig Grower Diet; LabDiet) and water ad libitum. To prevent interference with recipient swine activities, extracorporeal circuit tubing was secured at the top of the custom enclosure. One unit of whole blood was collected from each donor swine and was stored at $4{ }^{\circ} \mathrm{C}$. A transfusion was given to the recipient swine if hemoglobin decreased below $4 \mathrm{~g} / \mathrm{dL}$.

\section{Blood Analysis}

Before recovery of recipient swine from anesthesia, blood samples were collected from an auricular arterial line. Following recovery from anesthesia, blood samples were collected every 12 hours from a central venous line placed in the left external jugular vein. Hemogas analysis was performed using a point-of-care blood analysis system (epoc; Siemens Healthineers). Complete blood counts, basic metabolic panels, liver function tests, and coagulation panels were performed by a diagnostic laboratory service (Antech Diagnostics). Mycophenolate levels were not measured, but FK506 (tacrolimus) levels were measured (Architect System; Abbott). Inflammatory cytokines (granulocyte-macrophage colony-stimulating factor, interferon gamma, interleukin (IL) $1 \alpha$, IL-1 $\beta$, IL-1ra, IL-2, IL-4, IL6 , IL-8, IL-10, IL-12, IL-18, and tumor necrosis factor alpha) in recipient swine serum were quantified in triplicate by multiplex cytokine array (Discovery Assay Pig Cytokine Array; Eve Technologies).

\section{Extracorporeal Lung Monitoring}

Blood samples were collected from sample ports at the pulmonary artery and pulmonary veins every 12 hours throughout the duration of normothermic support. Arterial oxygen tension/inspired oxygen fraction and dynamic compliance $\left(\mathrm{C}_{\mathrm{dyn}}=\right.$ tidal volume/(peak inspiratory pressure - positive end-expiratory pressure)) were calculated at baseline and every 24 hours. Lung weight was obtained every 24 hours using a scale (Denver Instrument Company) housed inside the organ chamber. Gross photographs of extracorporeal lungs were acquired using a high-definition camera (Hero 5; GoPro), and radiographs were acquired using a portable unit (PXP-16HF; United Radiology Systems) with images captured at $2.2 \mathrm{mAs}$ and $90 \mathrm{kVp}$. Thermographs of extracorporeal lungs were acquired using an infrared camera (T430sc; FLIR).

\section{Bronchoscopy and Bronchoalveolar Lavage Fluid Analysis}

Bronchoscopic assessment of the airways was performed, and bronchographs were obtained at baseline and every 24 hours. Bronchoalveolar (BAL) fluid samples were collected by introducing a $3.8 \mathrm{~mm}$ flexible bronchoscope (aScope 3; Ambu) into subsegmental bronchi of the left and right lower lobes of extracorporeal lungs and injecting sterile normal saline $(5 \mathrm{~mL})$ with subsequent aspiration and collection of BAL fluid in a sterile specimen trap. BAL fluid samples were centrifuged at $3500 \mathrm{rpm}$ for 10 minutes at $4{ }^{\circ} \mathrm{C}$. Supernatants were snap frozen in liquid nitrogen and stored at $-80^{\circ} \mathrm{C}$ until further processing. Inflammatory cytokines (granulocyte-macrophage colony-stimulating factor, 
interferon gamma, IL-1 $\alpha$, IL-1 $\beta$, IL-1ra, IL-2, IL-4, IL-6, IL-8, IL-10, IL-12, IL-18, and tumor necrosis factor alpha) in BAL fluid of extracorporeal lungs were quantified in triplicate by multiplex cytokine array (Discovery Assay Pig Cytokine Array; Eve Technologies).

\section{Extracorporeal Lung Tissue Sampling}

A surgical stapler (GIA Auto Suture; Covidien) was used to collect tissue samples from extracorporeal lungs at baseline and every 24 hours. Tissue sampling locations were predetermined using randomization software (randomizer. org) (Figure E4, $A$ and $B$ ). Tissue samples were immediately fixed in cold phosphate-buffered $4 \%$ paraformaldehyde for 24 to 48 hours, embedded in paraffin, and sectioned at $5-\mu \mathrm{m}$ thickness. All sections were stained for hematoxylin and eosin and Movat's pentachrome by histology services in the Department of Molecular Pathology at Columbia University Medical Center. All sections were examined under light microscopy and imaged with a slide scanner (SCN400; Leica).

\section{Recipient Lung Tissue Sampling}

After each experiment the recipient lungs were procured via a median sternotomy, and a surgical stapler (GIA Auto Suture) was used to collect tissue samples from recipient lungs from all 5 lobes. Tissue samples were processed in a similar manner to extracorporeal lungs described above.

Transmission electron microscopy. Lung tissue samples were fixed with $2.5 \%$ glutaraldehyde, $4 \%$ paraformaldehyde, and $0.02 \%$ picric acid in $0.1 \mathrm{M} \mathrm{Na-cacodylate} \mathrm{buffer}$ (pH 7.2). Samples were then postfixed with $1 \%$ osmium tetroxide in Sorenson's buffer for 1 hour, dehydrated, and embedded in Lx-112 (Ladd Research Industries). Sections (thickness, $60 \mathrm{~nm}$ ) were prepared using an ultramicrotome, stained with uranyl acetate and lead citrate, and examined with an electron microscope (JEM-1200 EXII; JEOL). Images were captured with a digital camera (ORCA-HR; Hamamatsu Photonics).

Histopathologic review. An experienced lung transplant pathologist blinded to the study protocol performed all histopathologic review that included evaluation of lung tissue samples from upper, middle, and lower lobes. A previously reported lung injury scoring rubric (Figure $\mathrm{E} 4, C$ ) was used to assess the degree of lung injury. ${ }^{\mathrm{E} 1, \mathrm{E} 2}$

Immunohistochemical staining. Following deparaffinization, lung sections were subjected to boiling citrate buffer (pH 6.0) for antigen retrieval and blocked with $10 \%$ normal donkey serum in phosphate-buffered saline for 2 hours at room temperature. Next, primary antibodies (VE-cadherin: Abcam, ab232880; alpha smooth muscle actin: Abcam, ab5694) were diluted 1:200 and incubated for 2 hours. Secondary antibody (Abcam, ab150077) was diluted 1:300 and incubated for 2 hours at room temperature. Sections were subsequently mounted, and images were obtained using a fluorescent microscope (FSX100; Olympus).

Bronchoresponsiveness. Airway smooth muscle tone in extracorporeal lungs was assessed after 4 days of normothermic support by intratracheal delivery of nebulized methacholine chloride $(4 \mathrm{mg})$. Changes in peak inspiratory pressures were recorded for 1 minute before and 5 minutes after delivery of methacholine chloride.

Cell viability assay. To assess cell viability in the parenchyma of extracorporeal lungs, carboxyfluorescein succinimidyl ester (eBioscience) was reconstituted in dimethyl sulfoxide at a concentration of $1.06 \mathrm{M}$. After 4 days of normothermic support, carboxyfluorescein succinimidyl ester was delivered via flexible bronchoscope into distal regions of left and right lower lobes of extracorporeal lungs and incubated for 15 minutes. Lung tissue samples were then collected, washed 5 times with phosphate-buffered saline, fixed in cold phosphate-buffered $4 \%$ paraformaldehyde for 48 hours, embedded, deparaffinized, mounted, and imaged using a fluorescence microscope (FSX100).

Metabolic activity assay. To assess cellular metabolism in extracorporeal lungs, tissue samples were collected from the parenchyma at baseline, day 2 , and day 4 of normothermic support. Tissue samples (volume: $250 \mu \mathrm{L}$; triplicates) were finely minced, gently homogenized, and placed in a 96-well plate. Cell metabolism assay reagent (Alamar Blue; ThermoFisher) was diluted 1:10 in DMEM supplemented with $10 \%$ fetal bovine serum, and $100 \mu \mathrm{L}$ Alamar Blue reagent was added to wells containing lung sample homogenates. ${ }^{\mathrm{E} 3}$ Alamar Blue reagent alone $(100 \mu \mathrm{L})$ was added to wells containing no lung homogenate (negative controls). The multiwell plate was protected from light and incubated at $37^{\circ} \mathrm{C}$ with gentle shaking for 2 hours. Following incubation, absorbance was measured at $570 \mathrm{~nm}$ and normalized to absorbance at $600 \mathrm{~nm}$. To obtain a benchmark comparison of metabolic activity to healthy swine lungs in vivo, fresh lung tissues were collected from healthy swine immediately following thoracotomy, and metabolic activity assays were performed on both extracorporeal swine lung tissue samples and fresh healthy swine lung tissue samples.

\section{E-References}

E1. O'Neill JD, Guenthart BA, Kim J, Chicotka S, Queen D, Fung K, et al. Crosscirculation for extracorporeal support and recovery of the lung. Nat Biomed Eng. 2017;1:0037.

E2. Guenthart BA, O'Neill JD, Kim J, Queen D, Chicotka S, Fung K, et al. Regeneration of severely damaged lungs using an interventional cross-circulation platform. Nat Commun. 2019;10:1985.

E3. Rampersad SN. Multiple applications of alamar blue as an indicator of metabolic function and cellular health in cell viability bioassays. Sensors. 2012; 12:12347.

E4. Roman M, Gjorgjimajkoska O, Neil D, Nair S, Colah S, Parmar J, et al. Comparison between cellular and acellular perfusates for ex vivo lung perfusion in a porcine model. J Heart Lung Transplant. 2015;34:978-87. 
E5. Jung K, Renukaradhya GJ, Alekseev KP, Fang Y, Tang Y, Saif LJ. Porcine reproductive and respiratory syndrome virus modifies innate immunity and alters disease outcome in pigs subsequently infected with porcine respiratory coronavirus: implications for respiratory viral co-infections. J Gen Virol. 2009; 90(Pt 11):2713-23.

E6. Inci I, Ampollini L, Arni S, Jungraithmayr W, Inci D, Hillinger S, et al. Ex vivo reconditioning of marginal donor lungs injured by acid aspiration. J Heart Lung Transplant. 2008;27:1229-36.

E7. Guo B, Lager KM, Henningson JN, Miller LC, Schlink SN, Kappes MA, et al Experimental infection of United States swine with a Chinese highly pathogenic strain of porcine reproductive and respiratory syndrome virus. Virology. 2013;435:372-84.

E8. Nakajima D, Liu M, Ohsumi A, Kalaf R, Iskender I, Hsin M, et al. Lung lavage and surfactant replacement during ex vivo lung perfusion for treatment of gastric acid aspiration-induced donor lung injury. J Heart Lung Transplant. 2017;36:577-85.

E9. Iskender I, Cosgun T, Arni S, Trinkwitz M, Fehlings S, Yamada Y, et al. Cytokine filtration modulates pulmonary metabolism and edema formation during ex vivo lung perfusion. J Heart Lung Transplant. 2018;37:283-91.

E10. Adrian K, Skogby M, Gatzinsky V, Friberg L-G, Mellgren K. Procedureinduced inflammation and endothelial cell activation in an artificially ventilated and circulated porcine double-lung model. Artif Organs. 2006;30: 922-8.

E11. Protti A, Maraffi T, Milesi M, Votta E, Santini A, Pugni P, et al. Role of strain rate in the pathogenesis of ventilator-induced lung edema. Crit Care Med. 2016; 44:e838-45.
E12. Renukaradhya GJ, Alekseev K, Jung K, Fang Y, Saif LJ. Porcine reproductive and respiratory syndrome virus-induced immunosuppression exacerbates the inflammatory response to porcine respiratory coronavirus in pigs. Viral Immunol. 2010;23:457-66.

E13. Aboelnazar NS, Himmat S, Hatami S, White CW, Burhani MS, Dromparis P, et al. Negative pressure ventilation decreases inflammation and lung edema during normothermic ex-vivo lung perfusion. J Heart Lung Transplant. 2018;37: 520-30.

E14. Kakishita T, Oto T, Hori S, Miyoshi K, Otani S, Yamamoto S, et al. Suppression of inflammatory cytokines during ex vivo lung perfusion with an adsorbent membrane. Ann Thorac Surg. 2010;89:1773-9.

E15. LaPar DJ, Laubach VE, Emaminia A, Crosby IK, Hajzus VA, Sharma AK, et al Pretreatment strategy with adenosine A2A receptor agonist attenuates reperfusion injury in a preclinical porcine lung transplantation model. J Thorac Cardiovasc Surg. 2011;142:887-94.

E16. Mulloy DP, Stone ML, Crosby IK, Lapar DJ, Sharma AK, Webb DV, et al. Ex vivo rehabilitation of non-heart-beating donor lungs in preclinical porcine model: delayed perfusion results in superior lung function. J Thorac Cardiovasc Surg. 2012;144:1208-16.

E17. Khalifé-Hocquemiller T, Sage E, Dorfmuller P, Mussot S, Le Houérou D, Eddahibi S, et al. Exogenous surfactant attenuates lung injury from gastricacid aspiration during ex vivo reconditioning in pigs. Transplant J. 2014;97: 413-8.

E18. Yamada Y, Iskender I, Arni S, Hillinger S, Cosgun T, Yu K, et al. Ex vivo treatment with inhaled $\mathrm{N}$-acetylcysteine in porcine lung transplantation. J Surg Res. 2017:218:341-7. 


\begin{tabular}{lr} 
Extracorporeal circuit parameters & Target range \\
\hline PA pressure $(\mathrm{mm} \mathrm{Hg})$ & $<20$ \\
PV pressure $(\mathrm{mm} \mathrm{Hg})$ & $3-5^{\star}$ \\
Transpulmonary pressure gradient $(\mathrm{mm} \mathrm{Hg})$ & $5-15$ \\
\hline
\end{tabular}

A
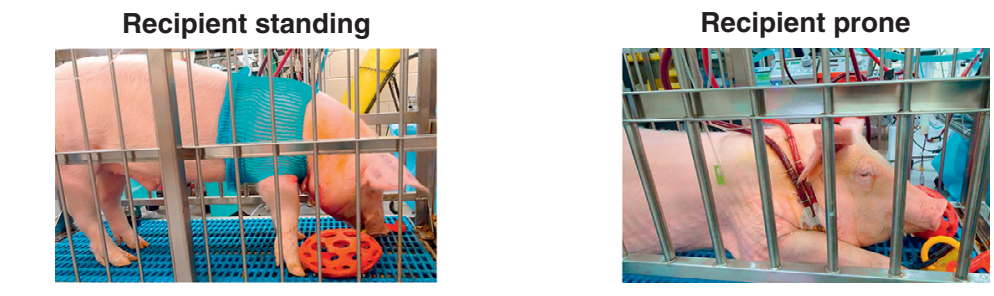

B

Recipient position

\begin{tabular}{lcr} 
Heights of circuit elements $(\mathrm{cm})$ & Standing & Prone \\
\hline $\mathrm{h}_{\text {lung }}$ & $86 \pm 4$ & $30 \pm 4$ \\
$\mathrm{~h}_{\text {recipient }}$ & $76 \pm 2$ & $20 \pm 2$ \\
$\mathrm{~h}_{\text {pump }}$ & $20 \pm 1$ & $20 \pm 1$ \\
$\mathrm{~h}_{\text {enclosure floor }}$ & 0 & 0 \\
\hline
\end{tabular}

C

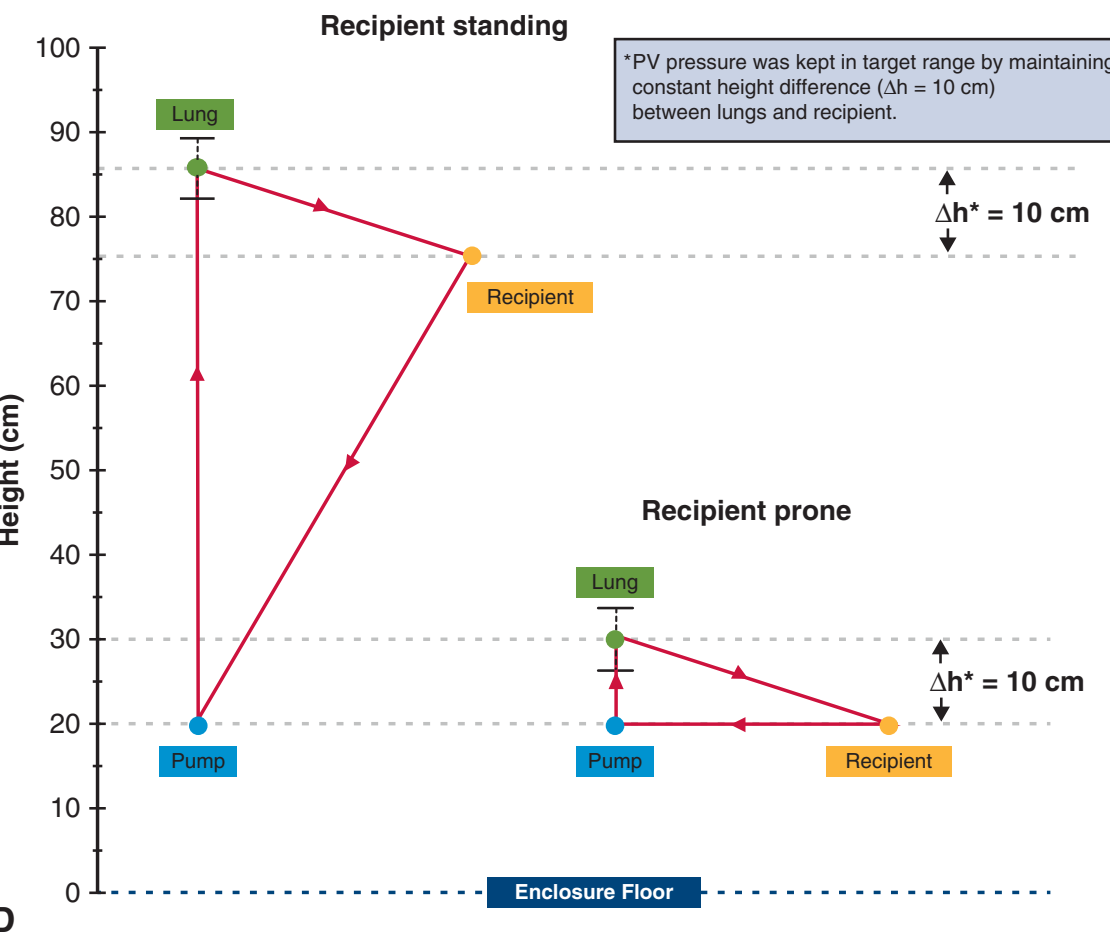

FIGURE E1. Maintenance of extracorporeal circuit parameters by height adjustments of extracorporeal lungs in response to changes in recipient swine position. A, Target ranges of extracorporeal circuit parameters. B, Representative photographs of recipient positions: upright and prone. C, Heights of circuit elements corresponding to recipient position. D, Extracorporeal circuit diagrams demonstrating changes in extracorporeal lung height corresponding to changes in recipient position in order to maintain target circuit parameters. $P A$, Pulmonary artery; $P V$, pulmonary vein. 


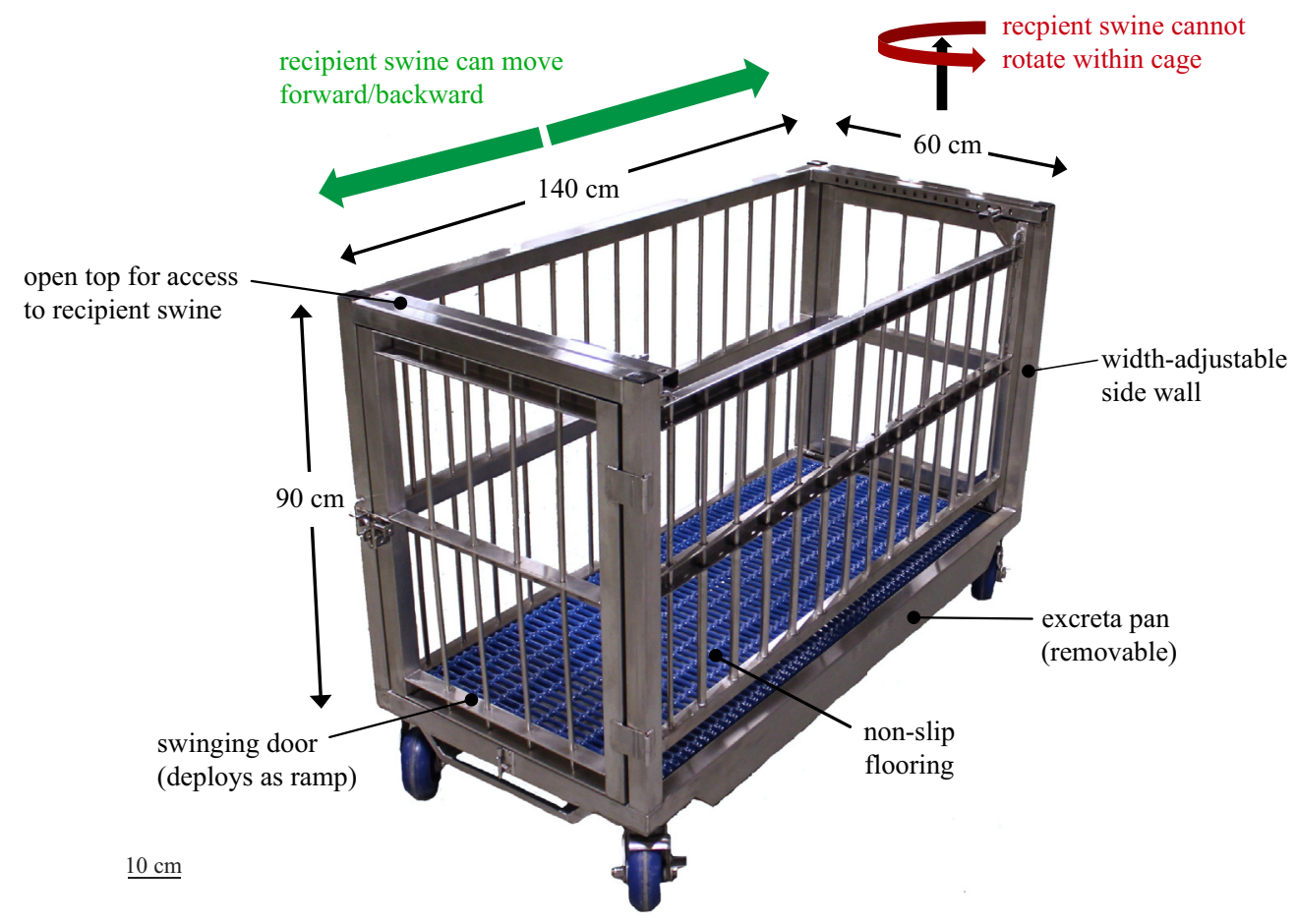

FIGURE E2. Custom enclosure for recipient swine. Stainless steel enclosure featured width-adjustable sidewall to ensure swine recipients remained comfortable and secure throughout multi-day procedures. The open top of the enclosure enabled easy access to recipient swine, cannula site, and circuit components. Immediately following recipient feeding, urination, and defecation, the excreta pan was removed, thoroughly cleaned, and replaced to minimize the presence of waste in the custom enclosure. 


\section{Baseline}

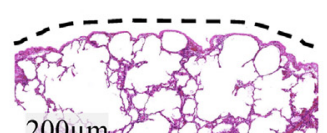

A $200 \mu m x^{2}$

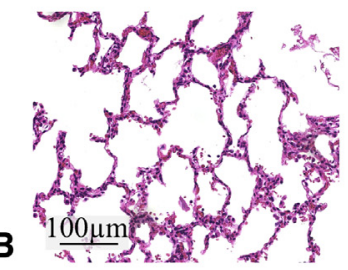

C
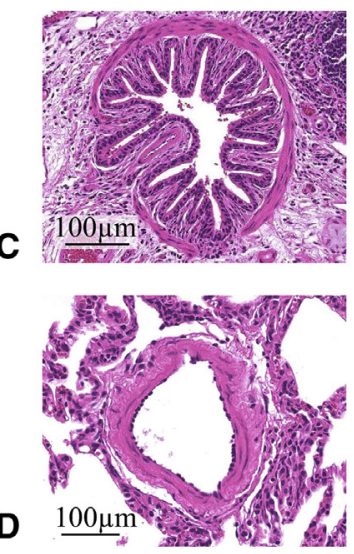

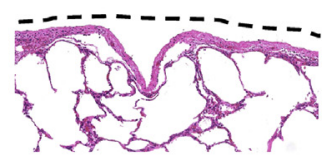

Day 1

Day 2
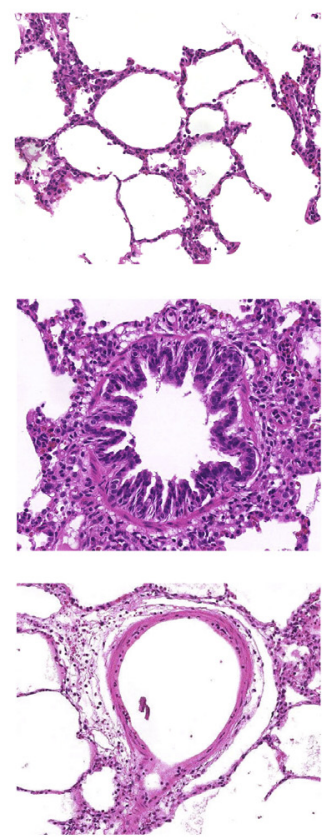

Day 3
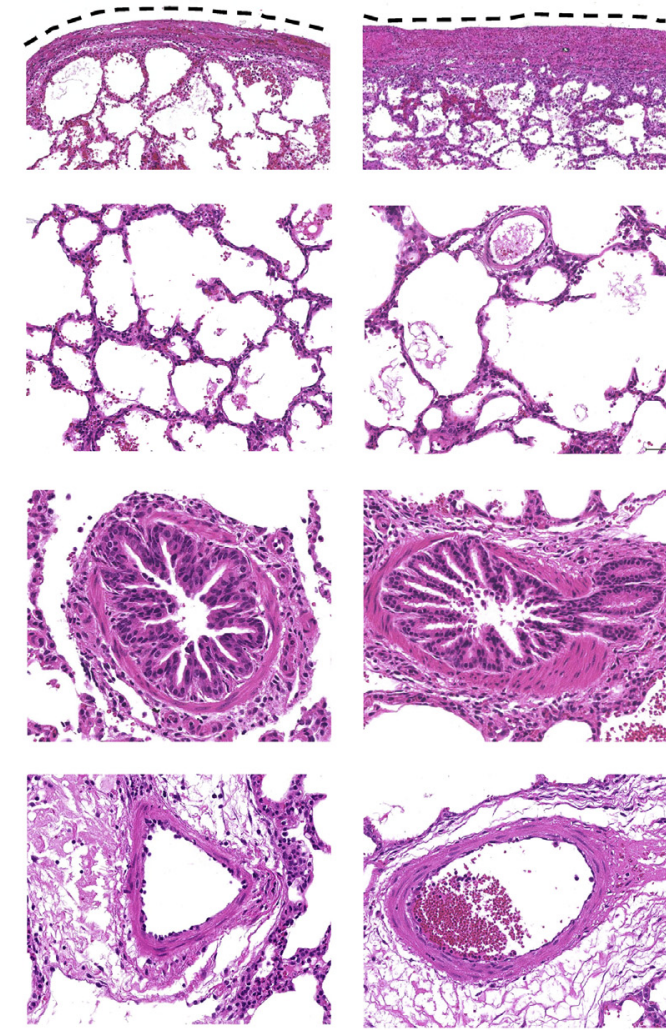

Day 4
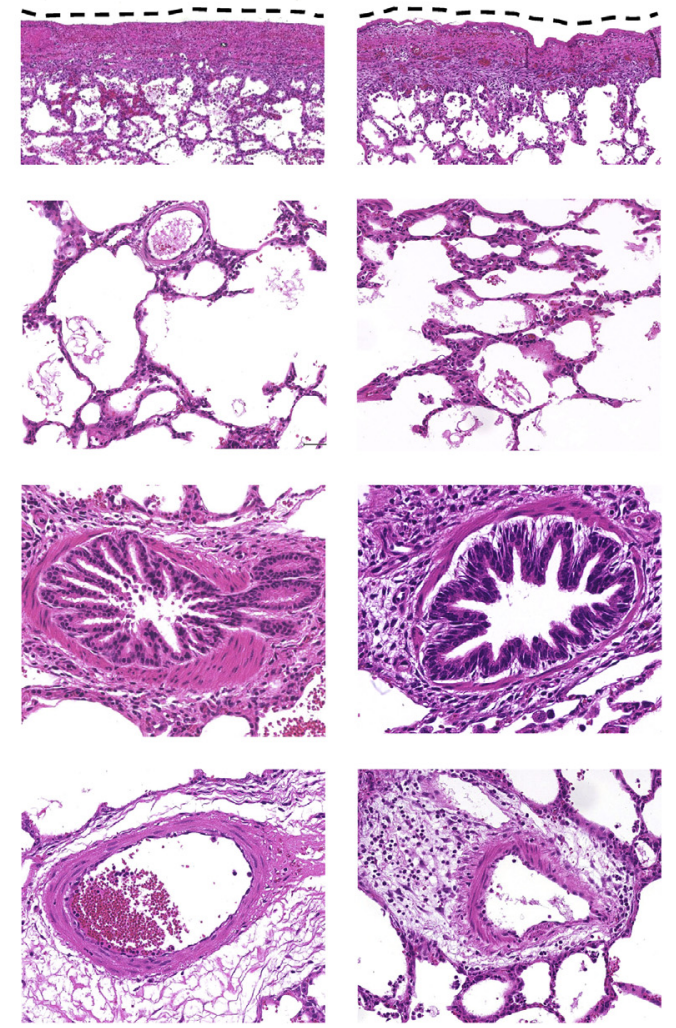

FIGURE E3. Histologic evaluation of the upper lobes of extracorporeal lungs throughout 4 days of normothermic support. A, Subpleural regions. B, Parenchyma. C, Pulmonary airways. D, vessels. Dotted lines outline surface of visceral pleura. 


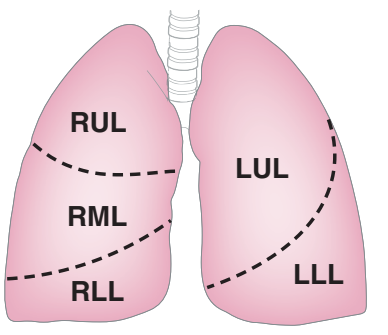

A

\begin{tabular}{|c|c|c|c|c|}
\hline Score & 0 & 1 & 2 & 3 \\
\hline Airway PMN / hpf ${ }^{1}$ & 0 & $1-25$ & $26-50$ & $>50$ \\
\hline Alveolar PMN / $\mathrm{hpf}^{2}$ & 0 & $1-25$ & $26-50$ & $>50$ \\
\hline Alveolar edema (\%) ${ }^{3}$ & $<5$ & $6-25$ & $26-50$ & $>50$ \\
\hline Interstitial infiltrate / $\mathrm{hpf}^{4}$ & none & $<50$ & $50-100$ & $>100$ \\
\hline Interstitial edema ${ }^{5}$ & 0 & $1 \mathrm{x}$ width vessel media & $\geq 2 x$ width vessel media & - \\
\hline
\end{tabular}

1 Airway PMN: \% bronchi and bronchioles containing any neutrophils

2 Alveolar PMN: \% alveoli more than half-filled with neutrophils

3 Alveolar edema: \% alveoli with edema

4 Interstitial infiltrate: lymphocytes/neutrophils in interstitium around vessels and airways and in alveolar septa and pleura

5 Interstitial edema: perivascular and peribronchial spaces expanded with edematous fluid

C

FIGURE E4. Randomized lung sampling and scoring rubric of lung injury score. A, Lung map used for randomized tissue sampling showing lungs were divided into 5 lobes. B, Tissue sample locations at each time point. Sample bias was avoided by predetermining tissue sampling location before the start of all experiments. C, Scoring rubric of lung injury scores. $R U L$, Right upper lobe; $R M L$, right middle lobe; $R L L$, right lower lobe; $L U L$, left upper lobe; $L L L$, left lower lobe; $P M N$, polymorphonuclear cells; $h p f$, high-power field.

Time (day) Tissue sampling location

\begin{tabular}{l|l}
0 & LUL, RLL, RML \\
1 & RUL, RLL, LLL \\
2 & LUL, LLL, RUL \\
3 & RUL, LLL, RML \\
4 & RUL, RML, RLL, LUL, LLL
\end{tabular}

B 

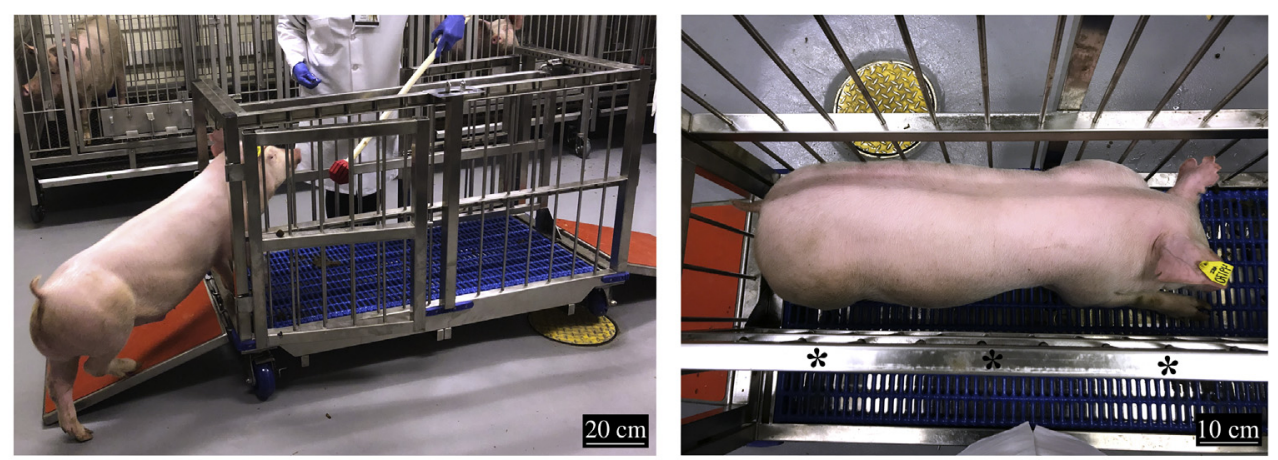

A

B
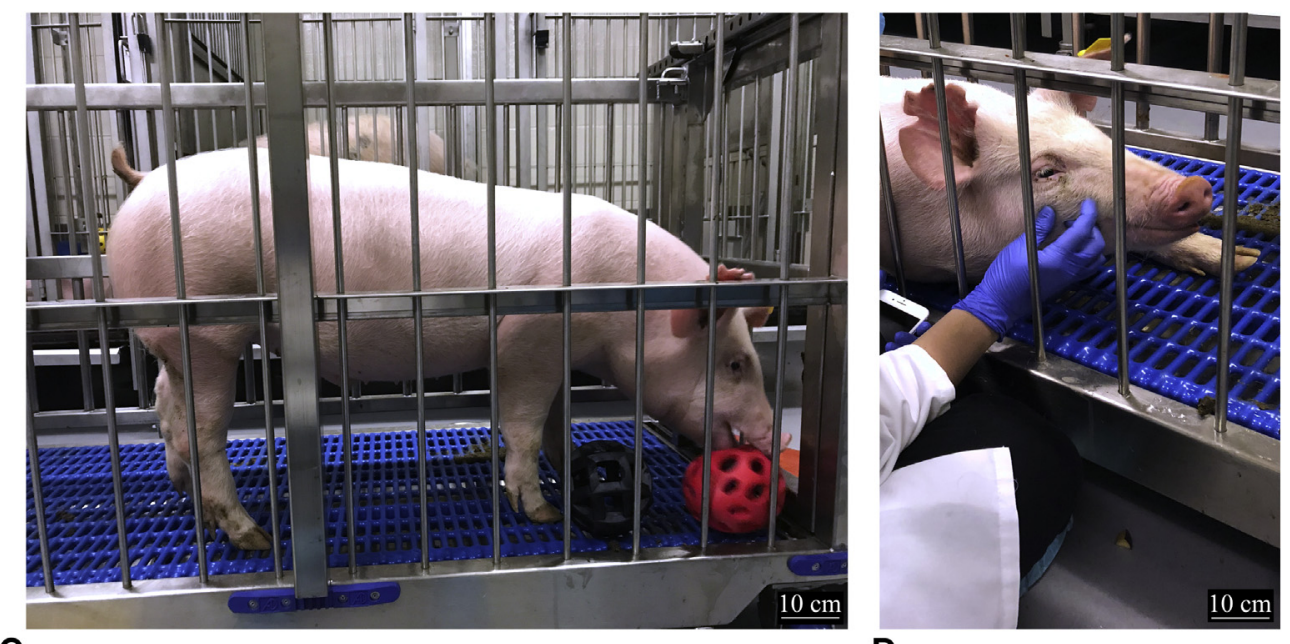

D

FIGURE E5. Preprocedure swine behavior training program. A, Target training with clicker (red) to encourage swine to enter custom enclosure. B, Throughout the behavior training program, recipient swine comfort and familiarity were maintained while the width of the side wall (stars) of the custom enclosure was incrementally decreased, limiting the ability of recipient swine to rotate during the procedure. C, Active enrichment was provided throughout behavior training. D, Positive reinforcement was provided throughout behavior training. 


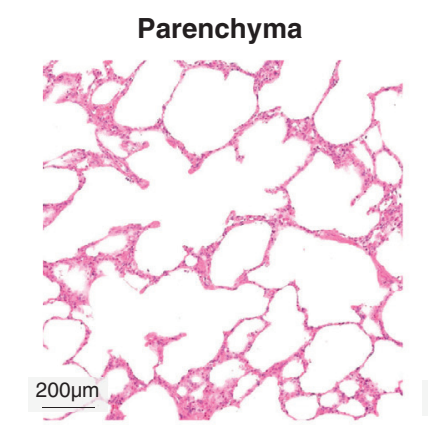

A

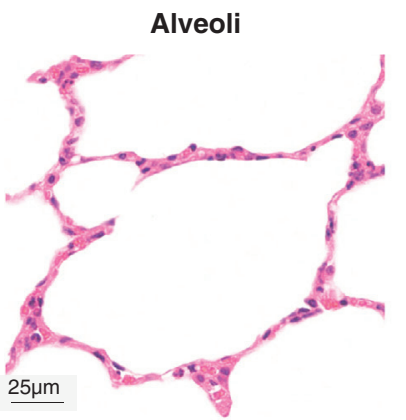

B

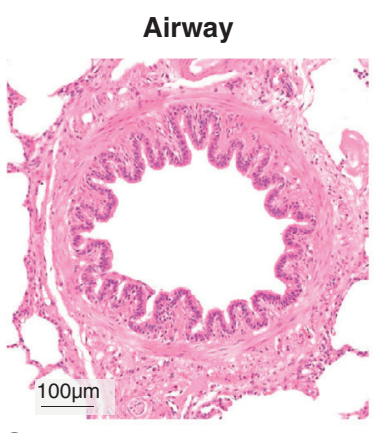

C

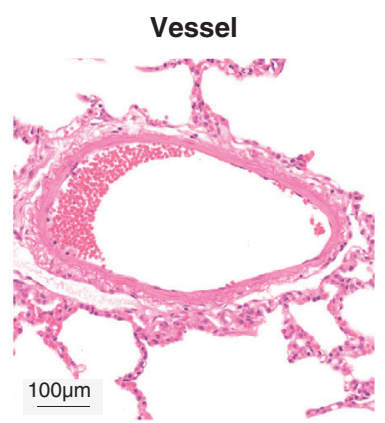

D

Lung injury scoring

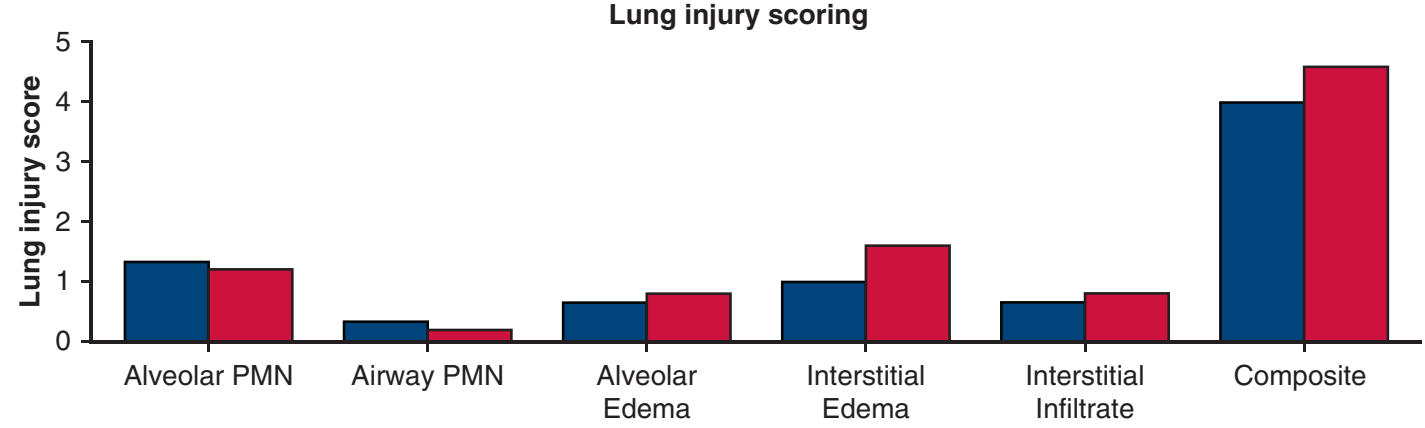

E

Recipient lungs at day 4 Extracorporeal lungs at day 4

FIGURE E6. Histologic evaluation by hematoxylin and eosin stain of recipient swine lung after 4 days of normothermic support. A, Low-power microscopy of parenchyma. B, High-power microscopy of alveoli. C, Airway. D, Vessels. E, Comparison of lung injury scores at day 4 of multiday support to lung injury scores of recipient lung at the end of the procedure. PMN, Polymorphonuclear cells. 
TABLE E1. Safety and stability of recipient swine during multiday extracorporeal lung support. Analysis of recipient swine vitals, hemogas, biochemistry, coagulation, and electrolytes throughout 4 days of cross-circulation

\begin{tabular}{|c|c|c|c|c|c|}
\hline \multirow[b]{2}{*}{ Parameter } & \multicolumn{5}{|c|}{ Time (d) } \\
\hline & $\mathbf{0}$ & 1 & 2 & 3 & 4 \\
\hline \multicolumn{6}{|l|}{ Vitals } \\
\hline Heart rate (bpm) & $88 \pm 9$ & $111 \pm 8$ & $113 \pm 51$ & $110 \pm 35$ & $140 \pm 42$ \\
\hline Systolic BP (mm Hg) & $97 \pm 14$ & $106 \pm 17$ & $155 \pm 9$ & $133 \pm 45$ & $89 \pm 37$ \\
\hline Temperature $\left({ }^{\circ} \mathrm{F}\right)$ & $97.3 \pm 1.9$ & $97.0 \pm 1.7$ & $96.2 \pm 2.8$ & $98.3 \pm 2.3$ & $98.5 \pm 1.0$ \\
\hline \multicolumn{6}{|l|}{ Hemogas } \\
\hline $\mathrm{pH}$ & $7.36 \pm 0.02$ & $7.46 \pm 0.02$ & $7.45 \pm 0.04$ & $7.46 \pm 0.05$ & $7.37 \pm 0.08$ \\
\hline Oxygen tension $(\mathrm{mm} \mathrm{Hg}) *$ & $274 \pm 34$ & $45 \pm 23$ & $39 \pm 16$ & $30 \pm 2$ & $42 \pm 3$ \\
\hline $\begin{array}{l}\text { Carbon dioxide tension } \\
\quad(\mathrm{mm} \mathrm{Hg})\end{array}$ & $57 \pm 3.4$ & $46 \pm 3.3$ & $50 \pm 8.5$ & $47 \pm 8.7$ & $42 \pm 5.8$ \\
\hline Bicarbonate $(\mathrm{mmol} / \mathrm{L})$ & $32 \pm 1.2$ & $33 \pm 1.7$ & $39 \pm 15.9$ & $34 \pm 3.8$ & $30 \pm 3.0$ \\
\hline Lactate $(\mathrm{mmol} / \mathrm{L})$ & $1.42 \pm 0.49$ & $1.58 \pm 0.38$ & $1.08 \pm 0.27$ & $0.91 \pm 0.31$ & $3.1 \pm 0.14$ \\
\hline Glucose $(\mathrm{mg} / \mathrm{dL})$ & $112 \pm 77$ & $199 \pm 49$ & $127 \pm 33$ & $177 \pm 55$ & $159 \pm 38$ \\
\hline \multicolumn{6}{|l|}{ Biochemical analysis } \\
\hline WBC $\left(10^{9} / \mathrm{L}\right)$ & $26.1 \pm 19.6$ & $18.3 \pm 4.2$ & $12.3 \pm 6.4$ & $14.3 \pm 1.9$ & $9.5 \pm 5.2$ \\
\hline$\%$ Neutrophils & $47 \pm 11$ & $67 \pm 5$ & $53 \pm 14$ & $65 \pm 19$ & $68 \pm 12$ \\
\hline$\%$ Reticulocytes & $1 \pm 1$ & $1 \pm 1$ & $1 \pm 1$ & $1 \pm 1$ & $3 \pm 1$ \\
\hline Platelets $\left(10^{9} / \mathrm{L}\right)$ & $423 \pm 222.4$ & $307 \pm 47.7$ & $203 \pm 97.1$ & $118 \pm 53.8$ & $94 \pm 61.6$ \\
\hline $\mathrm{Hgb}(\mathrm{g} / \mathrm{dL})$ & $8.1 \pm 4.9$ & $7.6 \pm 0.8$ & $7.9 \pm 3.3$ & $4.9 \pm 0.6$ & $3.1 \pm 0.7$ \\
\hline Hct $(\%)$ & $27.2 \pm 16.6$ & $25.7 \pm 3.8$ & $20.8 \pm 3.9$ & $16.5 \pm 2.5$ & $10.7 \pm 2.3$ \\
\hline AST (U/L) & $47 \pm 16.9$ & $54 \pm 13.6$ & $49 \pm 13.4$ & $28 \pm 19.0$ & $37 \pm 3.5$ \\
\hline ALT (U/L) & $67 \pm 12.3$ & $61 \pm 23.2$ & $51 \pm 23.4$ & $40 \pm 1.2$ & $29 \pm 3.6$ \\
\hline Creatinine $(\mathrm{mg} / \mathrm{dL})$ & $1.33 \pm 0.26$ & $1.15 \pm 0.13$ & $1.01 \pm 0.14$ & $1.05 \pm 0.06$ & $1.74 \pm 0.58$ \\
\hline Tacrolimus (ng/mL) & $0.93 \pm 1.04$ & $13.37 \pm 9.06$ & $20.23 \pm 8.93$ & $22.37 \pm 8.89$ & $15.21 \pm 3.83$ \\
\hline Activated clotting time (s) & $296 \pm 83$ & $222 \pm 35$ & $225 \pm 65$ & $250 \pm 66$ & $239 \pm 47$ \\
\hline \multicolumn{6}{|l|}{ Electrolytes and other } \\
\hline Sodium (mmol/L) & $136 \pm 3.8$ & $141 \pm 2.4$ & $136 \pm 1.0$ & $136 \pm 1.0$ & $140 \pm 2.8$ \\
\hline Potassium (mmol/L) & $7.9 \pm 2.9$ & $6.3 \pm 2.5$ & $5.6 \pm 1.1$ & $5.6 \pm 1.1$ & $5.0 \pm 0.5$ \\
\hline Calcium (mg/dL) & $7.8 \pm 0.8$ & $7.2 \pm 1.1$ & $8.1 \pm 0.8$ & $8.1 \pm 0.8$ & $6.4 \pm 1.4$ \\
\hline Phosphate (mmol/L) & $11.4 \pm 2.0$ & $9.5 \pm 0.8$ & $9.2 \pm 0.6$ & $9.2 \pm 0.6$ & $10.9 \pm 0.6$ \\
\hline PTT (s) & $78.6 \pm 47.1$ & $78.1 \pm 40.3$ & $87.2 \pm 61.5$ & $64.1 \pm 56.0$ & $75.5 \pm 63.8$ \\
\hline PT (s) & $14.9 \pm 1.2$ & $14.5 \pm 0.8$ & $17.0 \pm 5.1$ & $10.5 \pm 7.0$ & $10.4 \pm 5.4$ \\
\hline
\end{tabular}


TABLE E2. Quantification of inflammatory cytokines in recipient swine serum

\begin{tabular}{|c|c|c|c|c|c|c|}
\hline \multirow{2}{*}{$\begin{array}{c}\text { Inflammatory cytokine (pg/ } \\
\text { mL) }\end{array}$} & \multicolumn{5}{|c|}{ Time (d) } & \multirow[b]{2}{*}{ Fold change* } \\
\hline & $\mathbf{0}$ & 1 & 2 & 3 & 4 & \\
\hline GM-CSF & $-\dagger$ & $-\dagger$ & $8.48 \pm 6.5$ & $32.68 \pm 57.2$ & $9.6 \pm 8.3$ & +9.6 \\
\hline $\mathrm{IFN} \gamma$ & $20.6 \pm 28.8$ & $15.3 \pm 30.5$ & $52.3 \pm 55.2$ & $736.1 \pm 1010.9$ & $396.3 \pm 477.5$ & +19.2 \\
\hline $\mathrm{IL}-1 \alpha$ & $2.6 \pm 2.3$ & $1.4 \pm 2.0$ & $2.4 \pm 4.0$ & $2.5 \pm 4.1$ & $1.9 \pm 2.0$ & -1.3 \\
\hline $\mathrm{IL}-1 \beta$ & $45.0 \pm 31.7$ & $37.7 \pm 35.3$ & $67.8 \pm 52.3$ & $169.7 \pm 182.7$ & $471.6 \pm 649.8$ & +10.4 \\
\hline IL-1ra & $930.0 \pm 932.7$ & $437.7 \pm 250.9$ & $294.1 \pm 229.9$ & $694.7 \pm 523.7$ & $65024.5 \pm 52388.8$ & +69 \\
\hline IL-2 & $8.5 \pm 13.0$ & $6.0 \pm 12.1$ & $16.1 \pm 29.6$ & $16.7 \pm 30.3$ & $0.9 \pm 1.6$ & -7.6 \\
\hline IL-4 & $55.3 \pm 65.8$ & $46.2 \pm 78.6$ & $38.8 \pm 63.2$ & $51.7 \pm 92.6$ & $13.4 \pm 11.6$ & -4.1 \\
\hline IL-6 & $59.6 \pm 110.7$ & $59.8 \pm 93.9$ & $76.4 \pm 124.6$ & $82.9 \pm 131.3$ & $184.4 \pm 126.1$ & +3.0 \\
\hline IL-8 & $56.3 \pm 47.5$ & $-\dagger$ & $9.5 \pm 11.6$ & $1.0 \pm 2.0$ & $3.8 \pm 3.4$ & -14.8 \\
\hline IL-10 & $46.4 \pm 40.7$ & $47.5 \pm 36.3$ & $57.8 \pm 55.8$ & $54.8 \pm 45.2$ & $129.9 \pm 33.9$ & +2.8 \\
\hline IL-12 & $672.2 \pm 257.4$ & $246.4 \pm 87.7$ & $375.7 \pm 149.0$ & $386.0 \pm 171.0$ & $625.4 \pm 107.2$ & -1.1 \\
\hline IL-18 & $673.3 \pm 665.6$ & $237.4 \pm 191.7$ & $178.0 \pm 187.2$ & $280.6 \pm 128.2$ & $320.7 \pm 84.6$ & -2.1 \\
\hline $\mathrm{TNF} \alpha$ & $12.9 \pm 21.9$ & $5.0 \pm 6.6$ & $8.1 \pm 6.4$ & $18.8 \pm 30.6$ & $22.9 \pm 14.7$ & +1.77 \\
\hline
\end{tabular}

Values are presented as mean \pm standard deviation. $G M$ - CSF, Granulocyte-macrophage colony-stimulating factor; $I F N \gamma$, interferon-gamma; $I L$, interleukin; $T N F \alpha$, tumor necrosis factor alpha. *Fold change $(-$ or +$)$ represents the change in cytokine concentration from day 0 to day 4 . $\dagger$ Dash indicates cytokine value below detectable assay range. 
TABLE E3. Comparison of reported values of serum inflammatory cytokines in swine lung and ex vivo lung perfusion (EVLP) studies

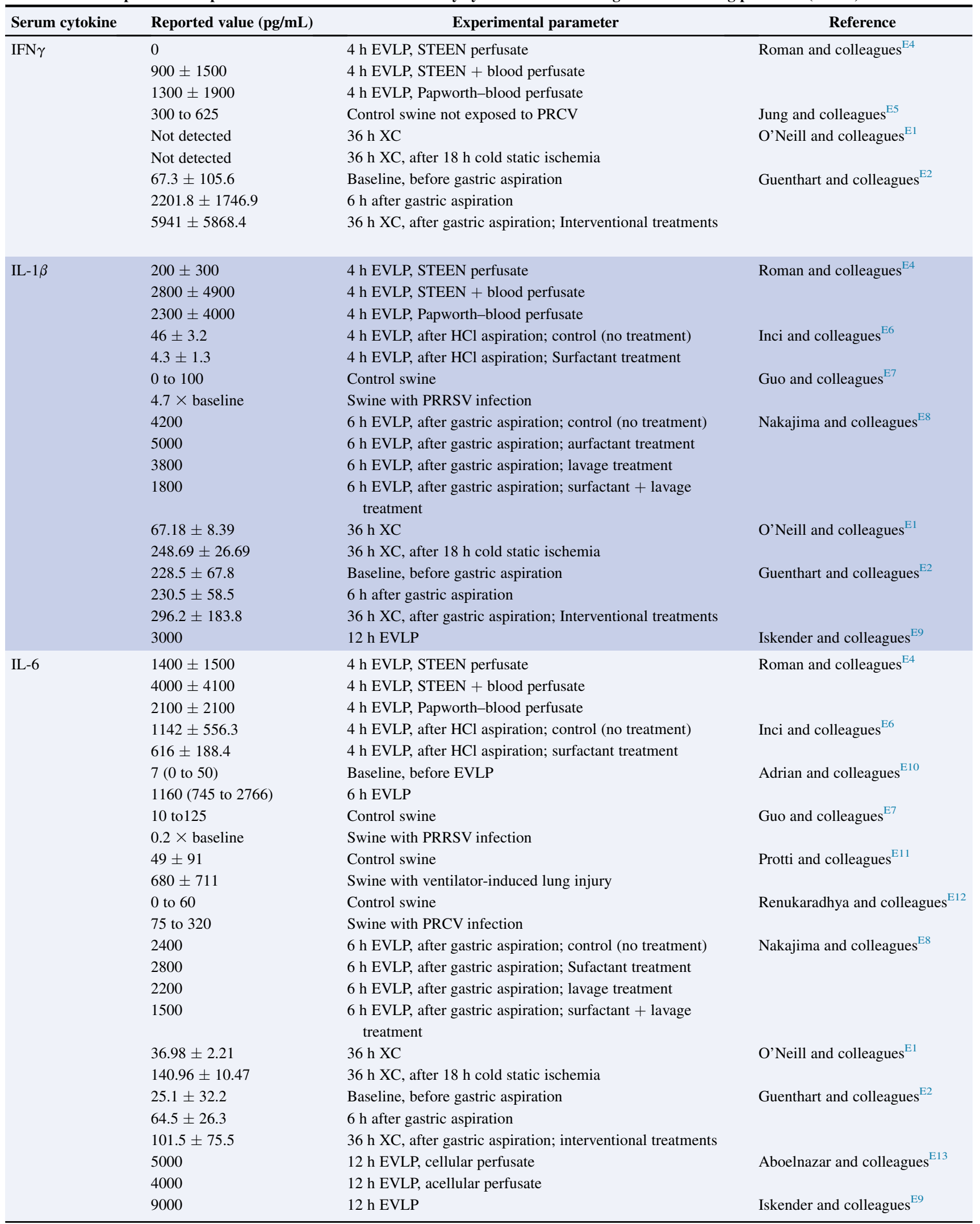


TABLE E3. Continued

\begin{tabular}{|c|c|c|c|}
\hline Serum cytokine & Reported value (pg/mL) & Experimental parameter & Reference \\
\hline \multirow[t]{20}{*}{ IL-8 } & $8700 \pm 1110$ & 4 h EVLP, STEEN perfusate & Roman and colleagues ${ }^{\mathrm{E} 4}$ \\
\hline & $5100 \pm 3400$ & $4 \mathrm{~h}$ EVLP, STEEN + blood perfusate & \\
\hline & $5600 \pm 6000$ & 4 h EVLP, Papworth-blood perfusate & \\
\hline & 6000 & $6 \mathrm{~h}$ EVLP & Kakishita and colleagues ${ }^{\mathrm{E} 14}$ \\
\hline & 12 to 18 & Control swine & Guo and colleagues ${ }^{\mathrm{E} 7}$ \\
\hline & $0.9 \times$ baseline & Swine with PRRSV infection & \\
\hline & $169 \pm 96$ & Control swine & Protti and colleagues ${ }^{\mathrm{E} 11}$ \\
\hline & $149 \pm 240$ & Swine with ventilator-induced lung injury & \\
\hline & 25,000 & $6 \mathrm{~h}$ EVLP, after gastric aspiration; control (no treatment) & Nakajima and colleagues ${ }^{\mathrm{E} 8}$ \\
\hline & 30,000 & $6 \mathrm{~h}$ EVLP, after gastric aspiration; surfactant treatment & \\
\hline & 18,000 & $6 \mathrm{~h}$ EVLP, after gastric aspiration; lavage treatment & \\
\hline & 8000 & $\begin{array}{l}6 \text { h EVLP, after gastric aspiration; surfactant + lavage } \\
\text { treatment }\end{array}$ & \\
\hline & $5.45 \pm 1.05$ & $36 \mathrm{~h} \mathrm{XC}$ & $\mathrm{O}^{\prime}$ Neill and colleagues ${ }^{\mathrm{E} 1}$ \\
\hline & $35.52 \pm 14.56$ & $36 \mathrm{~h} \mathrm{XC}$, after $18 \mathrm{~h}$ cold static ischemia & \\
\hline & $166.2 \pm 108.2$ & Baseline, before gastric aspiration & Guenthart and colleagues $^{\mathrm{E} 2}$ \\
\hline & $330.1 \pm 301.6$ & $6 \mathrm{~h}$ after gastric aspiration & \\
\hline & $309.6 \pm 241.8$ & $36 \mathrm{~h} \mathrm{XC}$, after gastric aspiration; interventional treatments & \\
\hline & 1400 & 12 h EVLP, cellular perfusate & Aboelnazar and colleagues ${ }^{\mathrm{E} 13}$ \\
\hline & 3000 & $12 \mathrm{~h}$ EVLP, acellular perfusate & \\
\hline & 12,000 & 12 h EVLP & Iskender and colleagues ${ }^{\mathrm{E} 9}$ \\
\hline \multirow[t]{15}{*}{ IL-10 } & $100 \pm 100$ & $4 \mathrm{~h}$ EVLP, STEEN perfusate & Roman and colleagues ${ }^{\mathrm{E} 4}$ \\
\hline & $600 \pm 500$ & 4 h EVLP, STEEN + blood perfusate & \\
\hline & $400 \pm 5000$ & $4 \mathrm{~h}$ EVLP, Papworth-blood perfusate & \\
\hline & $31(29$ to 49$)$ & Baseline, before EVLP & Adrian and colleagues ${ }^{\mathrm{E} 10}$ \\
\hline & $51(33$ to 91$)$ & $6 \mathrm{~h}$ EVLP & \\
\hline & $<4$ & Control swine & Protti and colleagues ${ }^{\mathrm{E} 11}$ \\
\hline & $<4$ & Swine with ventilator-induced lung injury & \\
\hline & 30 to 60 & Control swine & Renukaradhya and colleagues $^{\mathrm{E} 12}$ \\
\hline & 75 to 160 & Swine with PRCV infection & \\
\hline & $9.62 \pm 0.97$ & $36 \mathrm{~h} \mathrm{XC}$ & $\mathrm{O}^{\prime}$ Neill and colleagues ${ }^{\mathrm{E} 1}$ \\
\hline & $13.14 \pm 1.33$ & $36 \mathrm{~h} \mathrm{XC}$, after $18 \mathrm{~h}$ cold static ischemia & \\
\hline & $142.8 \pm 84.2$ & Baseline, before gastric aspiration & Guenthart and colleagues ${ }^{\mathrm{E} 2}$ \\
\hline & $149.1 \pm 84.3$ & $6 \mathrm{~h}$ after gastric aspiration & \\
\hline & $137.5 \pm 138.3$ & $36 \mathrm{~h} \mathrm{XC}$, after gastric aspiration; Interventional treatments & \\
\hline & 450 & 12 h EVLP & Iskender and colleagues ${ }^{\mathrm{E} 9}$ \\
\hline \multirow[t]{16}{*}{$\mathrm{TNF} \alpha$} & $500 \pm 700$ & $4 \mathrm{~h}$ EVLP, STEEN perfusate & Roman and colleagues ${ }^{\mathrm{E} 4}$ \\
\hline & $2400 \pm 2600$ & 4 h EVLP, STEEN + blood perfusate & \\
\hline & $1600 \pm 1600$ & 4 h EVLP, Papworth-blood perfusate & \\
\hline & 3800 & $6 \mathrm{~h}$ EVLP & Kakishita and colleagues ${ }^{\mathrm{E} 14}$ \\
\hline & 12 to 20 & Control swine & Guo and colleagues ${ }^{\mathrm{E} 7}$ \\
\hline & $3 \times$ baseline & Swine with PRRSV infection & \\
\hline & $113 \pm 47$ & Control swine & Protti and colleagues ${ }^{\mathrm{E} 11}$ \\
\hline & $83 \pm 59$ & Swine with ventilator-induced lung injury & \\
\hline & $7.67 \pm 1.12$ & $36 \mathrm{~h} \mathrm{XC}$ & $\mathrm{O}^{\prime}$ Neill and colleagues ${ }^{\mathrm{E} 1}$ \\
\hline & $84.76 \pm 14.41$ & $36 \mathrm{~h} \mathrm{XC}$, after $18 \mathrm{~h}$ cold static ischemia & \\
\hline & $26.6 \pm 16.9$ & Baseline, before gastric aspiration & Guenthart and colleagues $^{\mathrm{E} 2}$ \\
\hline & $62.2 \pm 57.5$ & $6 \mathrm{~h}$ after gastric aspiration & \\
\hline & $180.7 \pm 124.1$ & $36 \mathrm{~h} \mathrm{XC}$, after gastric aspiration; Interventional treatments & \\
\hline & 2200 & $12 \mathrm{~h}$ EVLP, cellular perfusate & Aboelnazar and colleagues ${ }^{\mathrm{E} 13}$ \\
\hline & 2200 & $12 \mathrm{~h}$ EVLP, acellular perfusate & \\
\hline & 200 & $12 \mathrm{~h} \mathrm{EVLP}$ & Iskender and colleagues ${ }^{\mathrm{E} 9}$ \\
\hline
\end{tabular}

$\overline{I F N \gamma}$, Interferon gamma; $E V L P$, ex vivo lung perfusion; $P R C V$, porcine respiratory coronavirus; $X C$, cross-circulation; $I L$, interleukin; $H C l$, hydrochloric acid; $P R R S V$, porcine reproductive and respiratory syndrome virus; $T N F \alpha$, tumor necrosis factor alpha. 
TABLE E4. Quantification of functional parameters of extracorporeal lungs over 4 days of normothermic support

\begin{tabular}{lr}
\hline \multicolumn{1}{c}{ Parameter } & \multicolumn{1}{c}{ Result } \\
\hline Arterial oxygen tension/Inspired oxygen fraction & \\
$\quad(\mathrm{mm} \mathrm{Hg})$ & \\
Baseline & $439.4 \pm 227$ \\
Day 4 & $548.5 \pm 176$ \\
Change & $+109.1 \pm 51$ \\
Compliance $\left(\mathrm{mL} / \mathrm{cmH}_{2} \mathrm{O}\right)$ & \\
Baseline & $22.3 \pm 1.6$ \\
Day 4 & $20.0 \pm 1.0$ \\
Change & $-1.7 \pm 0.6$ \\
Peak inspiratory pressure (mm Hg) & \\
Baseline & $20.5 \pm 0.7$ \\
Day 4 & $25.0 \pm 4.2$ \\
Change & $-4.5 \pm 3.5$ \\
Lactate (mmol) & \\
Baseline & $1.4 \pm 0.5$ \\
Day 4 & $3.1 \pm 0.2$ \\
Change & $+1.7 \pm 0.3$ \\
pH & \\
Baseline & $7.36 \pm 0.02$ \\
Day 4 & $7.37 \pm 0.08$ \\
Change & $+0.01 \pm 0.06$ \\
\hline
\end{tabular}

Values are presented as mean \pm standard deviation. 
TABLE E5. Quantification of inflammatory cytokines in bronchoalveolar lavage (BAL) fluid of extracorporeal lungs

\begin{tabular}{|c|c|c|c|c|c|c|}
\hline \multirow{2}{*}{$\begin{array}{c}\text { Inflammatory cytokine } \\
(\mathrm{pg} / \mathrm{mL})\end{array}$} & \multicolumn{5}{|c|}{ Time (d) } & \multirow[b]{2}{*}{ Fold change } \\
\hline & $\mathbf{0}$ & 1 & 2 & 3 & 4 & \\
\hline GM-CSF & $11.1 \pm 10.9$ & $5.9 \pm 8.2$ & $2.1 \pm 4.6$ & $13.9 \pm 17.0$ & $28.1 \pm 25.1$ & +2.5 \\
\hline $\mathrm{IFN} \gamma$ & $104.9 \pm 192.5$ & $7.19 \pm 16.1$ & $2.1 \pm 4.7$ & $3.64 \pm 8.1$ & $-\dagger$ & -104.9 \\
\hline IL- $1 \alpha$ & $614.6 \pm 670.6$ & $35.4 \pm 16.1$ & $399.8 \pm 480.2$ & $115.0 \pm 144.0$ & $69.7 \pm 91.0$ & -8.8 \\
\hline IL- $1 \beta$ & $4831.3 \pm 5898.4$ & $1142.6 \pm 1065.1$ & $5772.9 \pm 5662.4$ & $3377.6 \pm 4347.7$ & $2161.5 \pm 2457.7$ & -2.2 \\
\hline IL-1ra & $4372.3 \pm 6104.8$ & $725.9 \pm 664.2$ & $5367.5 \pm 6231.2$ & $4096.5 \pm 4210.2$ & $3455.6 \pm 2516.0$ & -1.2 \\
\hline IL-2 & $4105.6 \pm 6311.4$ & $420.1 \pm 746.6$ & $4866.6 \pm 6665.0$ & $3057.3 \pm 4591.0$ & $1653.1 \pm 2858.3$ & -2.4 \\
\hline IL-4 & $4.1 \pm 9.2$ & $-\dagger$ & $4.4 \pm 9.8$ & $32.9 \pm 43.9$ & $22.7 \pm 39.4$ & +5.5 \\
\hline IL-6 & $94.7 \pm 117.8$ & $466.2 \pm 513.3$ & $719.6 \pm 859.3$ & $684.7 \pm 789.6$ & $472.1 \pm 817.7$ & +4.9 \\
\hline IL-8 & $17797.1 \pm 18704.1$ & $8382.1 \pm 6745.5$ & $17069.0 \pm 12276.2$ & $12260.3 \pm 12016.8$ & $10917.3 \pm 12690.8$ & -1.6 \\
\hline IL-10 & $6.1 \pm 1.7$ & $4.4 \pm 3.1$ & $4.0 \pm 3.6$ & $23.4 \pm 25.1$ & $21.7 \pm 27.8$ & +3.5 \\
\hline IL-12 & $66.6 \pm 92.7$ & $16.8 \pm 15.9$ & $24.5 \pm 32.9$ & $63.2 \pm 78.2$ & $53.9 \pm 83.7$ & -1.2 \\
\hline IL-18 & $710.4 \pm 959.6$ & $146.2 \pm 186.0$ & $581.2 \pm 779.8$ & $186.1 \pm 198.6$ & $130.0 \pm 215.5$ & -5.4 \\
\hline $\mathrm{TNF} \alpha$ & $704.1 \pm 965.4$ & $126.2 \pm 198.5$ & $573.2 \pm 786.9$ & $158.2 \pm 217.0$ & $126.3 \pm 218.7$ & -5.5 \\
\hline
\end{tabular}

Values are presented as mean \pm standard deviation of cytokine concentrations in BAL fluid collected from both left and right lungs. GM-CSF, Granulocyte-macrophage colonystimulating factor; $I F N \gamma$, interferon-gamma; $I L$, interleukin; $T N F \alpha$, tumor necrosis factor alpha. *Fold change represents the change (- or +$)$ in cytokine concentration from day 0 to day 4 . $†$ Cytokine level below detectable assay range. 
TABLE E6. Comparison of reported values of bronchoalveolar lavage fluid (BALF) inflammatory cytokines in swine lung and ex vivo lung perfusion (EVLP) studies

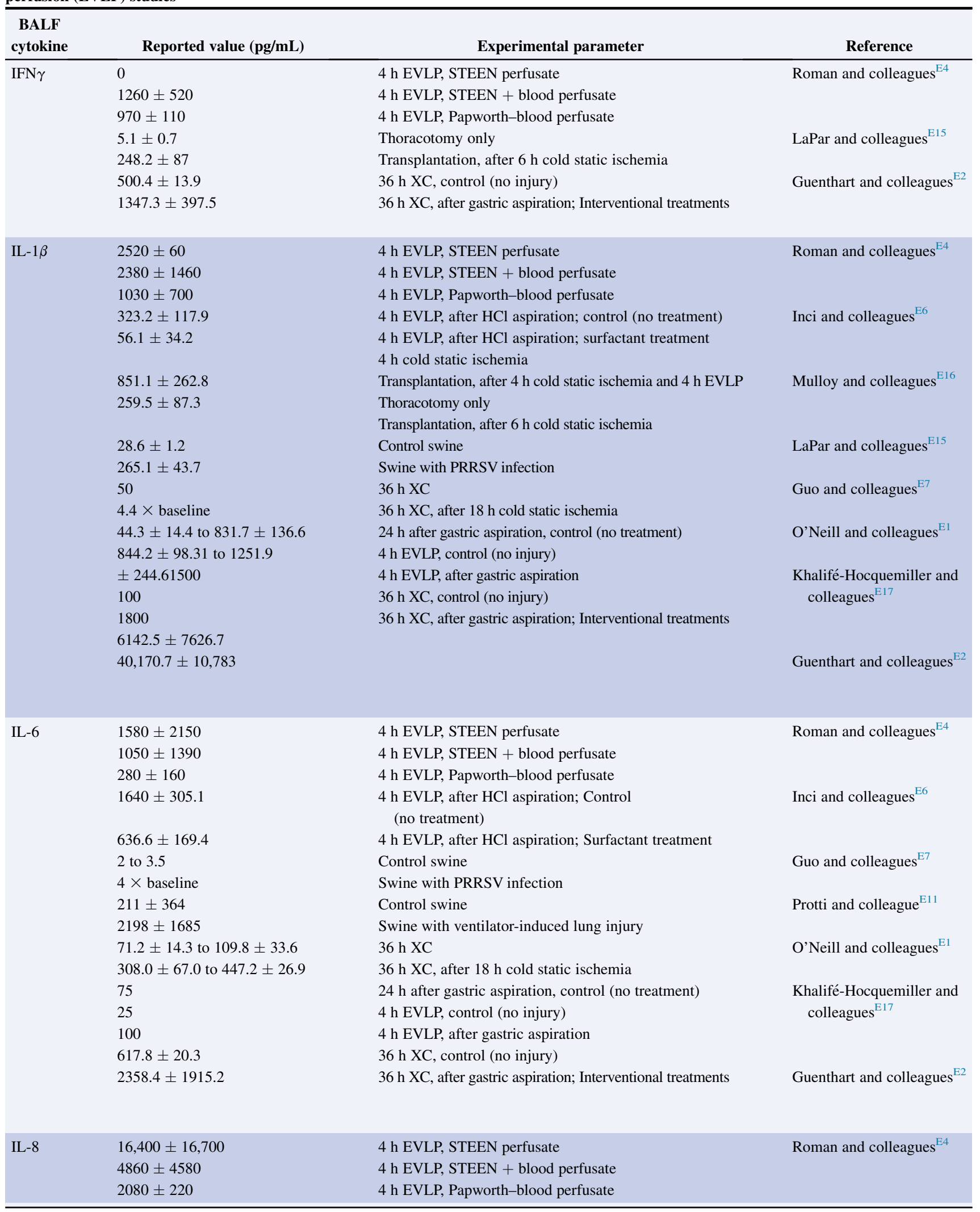


TABLE E6. Continued

\begin{tabular}{|c|c|c|c|}
\hline $\begin{array}{c}\text { BALF } \\
\text { cytokine }\end{array}$ & Reported value $(\mathrm{pg} / \mathrm{mL})$ & Experimental parameter & Reference \\
\hline & $1795.5 \pm 164.9$ & 4 h EVLP, after $\mathrm{HCl}$ aspiration; Control (no treatment) & Inci and colleagues ${ }^{\mathrm{E} 6}$ \\
\hline & & 4 h EVLP, after $\mathrm{HCl}$ aspiration; Surfactant treatment & \\
\hline & $659.5 \pm 166.5$ & $4 \mathrm{~h}$ cold static ischemia & \\
\hline & $531.5 \pm 331.4$ & Transplantation, after $4 \mathrm{~h}$ cold static ischemia and $4 \mathrm{~h}$ EVLP & Mulloy and colleagues ${ }^{\mathrm{E} 16}$ \\
\hline & $112.1 \pm 74.8$ & Thoracotomy only & \\
\hline & & Transplantation, after $6 \mathrm{~h}$ cold static ischemia & \\
\hline & $51.3 \pm 16.7$ & Control swine & LaPar and colleagues ${ }^{\mathrm{E} 15}$ \\
\hline & $222.4 \pm 41.4$ & Swine with PRRSV infection & \\
\hline & 25 to 35 & Control swine & Guo and colleagues ${ }^{\mathrm{E} 7}$ \\
\hline & $7.6 \times$ baseline & Swine with ventilator-induced lung injury & \\
\hline & $1569 \pm 1475$ & $36 \mathrm{~h} \mathrm{XC}$ & Protti and colleagues ${ }^{\mathrm{E} 11}$ \\
\hline & $725 \pm 1092$ & $36 \mathrm{~h} \mathrm{XC}$, after $18 \mathrm{~h}$ cold static ischemia & \\
\hline & $0.1 \pm 0.1$ to $3.0 \pm 1.0$ & $24 \mathrm{~h}$ after gastric aspiration, control (no treatment) & $\mathrm{O}^{\prime}$ Neill and colleagues ${ }^{\mathrm{E} 1}$ \\
\hline & $4.0 \pm 0.8$ to $5.47 \pm 1.8$ & $4 \mathrm{~h}$ EVLP, control (no injury) & \\
\hline & 10 & $4 \mathrm{~h}$ EVLP, after gastric aspiration & Khalifé-Hocquemiller and \\
\hline & 0.4 & $36 \mathrm{~h} \mathrm{XC}$, control (no injury) & colleagues $^{\mathrm{E} 17}$ \\
\hline & 11 & $36 \mathrm{~h} \mathrm{XC}$, after gastric aspiration; Interventional treatments & \\
\hline & $2529.6 \pm 77.3$ & $2 \mathrm{~h}$ EVLP, after $24 \mathrm{~h}$ cold static ischemia (control) & \\
\hline & $3188.4 \pm 169.2$ & & Guenthart and colleagues $^{\mathrm{E} 2}$ \\
\hline & $336.8 \pm 447.2$ to $3847.7 \pm 796.1$ & & \\
\hline & & & Yamada and colleagues $^{\mathrm{E} 18}$ \\
\hline IL-10 & $120 \pm 160$ & $4 \mathrm{~h}$ EVLP, STEEN perfusate & Roman and colleagues ${ }^{\mathrm{E} 4}$ \\
\hline & $90 \pm 120$ & 4 h EVLP, STEEN + blood perfusate & \\
\hline & $10 \pm 2$ & 4 h EVLP, Papworth-blood perfusate & \\
\hline & $2.7 \pm 0.8$ & Thoracotomy only & LaPar and colleagues ${ }^{\mathrm{E} 15}$ \\
\hline & $63.1 \pm 13.1$ & Transplantation, after $6 \mathrm{~h}$ cold static ischemia & \\
\hline & $<4$ & Control swine & Protti and colleagues ${ }^{\mathrm{E} 11}$ \\
\hline & $<4$ & Swine with ventilator-induced lung injury & \\
\hline & $3.4 \pm 1.7$ to $19.0 \pm 6.6$ & $36 \mathrm{~h} \mathrm{XC}$ & $\mathrm{O}^{\prime}$ Neill and colleagues ${ }^{\mathrm{E} 1}$ \\
\hline & $11.8 \pm 4.6$ to $14.1 \pm 1.4$ & $36 \mathrm{~h} \mathrm{XC}$, after $18 \mathrm{~h}$ cold static ischemia & \\
\hline & $11.1 \pm 9.7$ to $46.9 \pm 49.7$ & $36 \mathrm{~h} \mathrm{XC}$, control (no injury) & Guenthart and colleagues ${ }^{\mathrm{E} 2}$ \\
\hline & $11.1 \pm 9.7$ to $127.6 \pm 72.2$ & $36 \mathrm{~h} \mathrm{XC}$, after gastric aspiration; Interventional treatments & \\
\hline $\mathrm{TNF} \alpha$ & $170 \pm 220$ & $4 \mathrm{~h}$ EVLP, STEEN perfusate & Roman and colleagues ${ }^{\mathrm{E} 4}$ \\
\hline & $360 \pm 510$ & $4 \mathrm{~h}$ EVLP, STEEN + blood perfusate & \\
\hline & $80 \pm 50$ & 4 h EVLP, Papworth-blood perfusate & \\
\hline & $1050.2 \pm 353.8$ & $4 \mathrm{~h}$ cold static ischemia & Mulloy and colleagues ${ }^{\mathrm{E} 16}$ \\
\hline & $233.2 \pm 84.9$ & $\begin{array}{l}\text { Transplantation, after } 4 \mathrm{~h} \text { cold static ischemia and } 4 \mathrm{~h} \text { EVLP } \\
\text { Thoracotomy only }\end{array}$ & \\
\hline & $2.5 \pm 0.6$ & Transplantation, after $6 \mathrm{~h}$ cold static ischemia & LaPar and colleagues ${ }^{\mathrm{E} 15}$ \\
\hline & $25.9 \pm 4.6$ & Control swine & \\
\hline & 0.5 to 1.5 & Swine with PRRSV infection & Guo and colleagues ${ }^{\mathrm{E} 7}$ \\
\hline & $20.6 \times$ baseline & Control swine & \\
\hline & $202 \pm 252$ & Swine with ventilator-induced lung injury & Protti and colleagues ${ }^{\mathrm{E} 11}$ \\
\hline & $46 \pm 71$ & $36 \mathrm{~h} \mathrm{XC}$ & \\
\hline & $0.8 \pm 0.5$ to $2.7 \pm 0.8$ & $36 \mathrm{~h} \mathrm{XC}$, after $18 \mathrm{~h}$ cold static ischemia & $\mathrm{O}^{\prime}$ Neill and colleagues ${ }^{\mathrm{E} 1}$ \\
\hline & $53.8 \pm 13.3$ to $96.6 \pm 10.5$ & $36 \mathrm{~h} \mathrm{XC}$, control (no injury) & \\
\hline & $5.1 \pm 8.9$ to $32.5 \pm 16.2$ & $36 \mathrm{~h} \mathrm{XC}$, after gastric aspiration; Interventional treatments & Guenthart and colleagues $^{\mathrm{E} 2}$ \\
\hline & $5.1 \pm 8.9$ to $61.7 \pm 20.8$ & & \\
\hline
\end{tabular}

$\overline{I F N \gamma}$, Interferon gamma; $E V L P$, ex vivo lung perfusion; $X C$, cross-circulation; IL, interleukin; $H C l$, hydrochloric acid; $P R R S$, porcine reproductive and respiratory syndrome virus; $T N F \alpha$, tumor necrosis factor alpha. 
TABLE E7. Preprocedure training program for recipient swine. Before initiation of cross-circulation between extracorporeal lungs and recipient swine, preprocedure behavior training was conducted for 13 days. On day 14, cross-circulation was initiated and continued through day 18

\begin{tabular}{|c|c|}
\hline Day & Activity \\
\hline 0 & Arrival at animal housing facility where recipient swine were placed into standard housing enclosure. \\
\hline $1-2$ & $\begin{array}{l}\text { Initiation of target training in swine housing enclosure. Swine conditioned to touch nose to target when trainer used clicker. Behavior } \\
\text { reinforced with standard quality edible treats. Duration: } 1 \mathrm{~h} \text { daily. }\end{array}$ \\
\hline 3 & $\begin{array}{l}\text { Continuation of target training in housing enclosure. Introduction of custom enclosure within the standard housing enclosure area. } \\
\text { Duration: } 1 \mathrm{~h} \text {. }\end{array}$ \\
\hline 4 & $\begin{array}{l}\text { Progression of target training to encourage swine to enter custom enclosure. Duration: } 1 \mathrm{~h} \text {. } \\
\text { Swine placed into custom enclosure and transported to operating room. Duration: } 1 \mathrm{~h} \text {. }\end{array}$ \\
\hline $5-13$ & $\begin{array}{l}\text { Continuation of daily decreases in width of custom enclosure to inhibit swine from rotating within the cage while remaining } \\
\text { comfortable and allowing forward, backward, upward, and downward movements. Swine encouraged to remain within the } \\
\text { enclosure using high quality edible treats. Duration: } 2 \mathrm{~h} \text { daily. }\end{array}$ \\
\hline 14 & $\begin{array}{l}\text { Transportation to operating room where swine were anesthetized, cross-circulation was initiated, and swine were placed into custom } \\
\text { enclosure. }\end{array}$ \\
\hline $14-18$ & $\begin{array}{l}\text { Active enrichment during multiday extracorporeal organ support studies using manipulata (ie, toys), social interactions with } \\
\text { procedure personnel, and standard and high quality edible treats. }\end{array}$ \\
\hline
\end{tabular}

